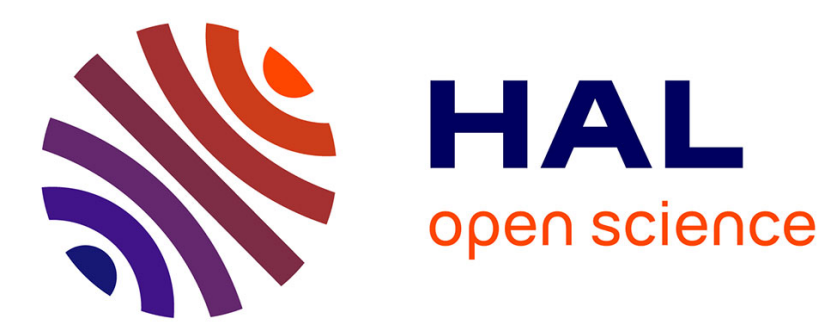

\title{
Inverse scattering at high energies for a classical particle in a long range force field
}

Alexandre Jollivet

\section{To cite this version:}

Alexandre Jollivet. Inverse scattering at high energies for a classical particle in a long range force field. 2014. hal-01063458v2

\section{HAL Id: hal-01063458 \\ https://hal.science/hal-01063458v2}

Preprint submitted on 16 Sep 2014

HAL is a multi-disciplinary open access archive for the deposit and dissemination of scientific research documents, whether they are published or not. The documents may come from teaching and research institutions in France or abroad, or from public or private research centers.
L'archive ouverte pluridisciplinaire HAL, est destinée au dépôt et à la diffusion de documents scientifiques de niveau recherche, publiés ou non, émanant des établissements d'enseignement et de recherche français ou étrangers, des laboratoires publics ou privés. 


\title{
Inverse scattering at high energies for a classical particle in a long range force field
}

\author{
Alexandre Jollivet*
}

September 16, 2014

\begin{abstract}
We define scattering data for the Newton equation in an electromagnetic field $(-\nabla V, B) \in C^{1}\left(\mathbb{R}^{n}, \mathbb{R}^{n}\right) \times C^{1}\left(\mathbb{R}^{n}, A_{n}(\mathbb{R})\right), n \geq 2$, that decay at infinity like $r^{-\alpha-1}$ for some $\alpha \in(0,1]$, where $A_{n}(\mathbb{R})$ is the space of $n \times n$ antisymmetric matrices. We provide their high energies asymptotics and we prove, in particular, that the scattering data at high energies uniquely determine the short range part of $(\nabla V, B)$ up to the knowledge of the long range part of $(\nabla V, B)$. Other asymptotic regimes are also considered. This paper extends similar results for a short range force field [Jollivet, 2009] or for a long range electric (or gravitational) field [Jollivet, 2013].
\end{abstract}

keywords: Newton equation; Long range electromagnetic field; Inverse scattering at high energies.

\section{Introduction}

Consider the multidimensional Newton equation in an external and static electromagnetic field:

$$
\ddot{x}(t)=F(x(t), \dot{x}(t)):=-\nabla V(x(t))+B(x(t)) \dot{x}(t),
$$

for $x(t) \in \mathbb{R}^{n}, t \in \mathbb{R}, n \geq 2$ where $\dot{x}(t)=\frac{d}{d t} x(t)$, and where $V \in C^{2}\left(\mathbb{R}^{n}, \mathbb{R}\right)$, $B(x)$ is the $n \times n$ real antisymmetric matrix with elements $B_{i, k}, 1 \leq i, k \leq n$, and where $B$ satisfies the closure condition

$$
\frac{\partial}{\partial x_{i}} B_{k, m}(x)+\frac{\partial}{\partial x_{m}} B_{i, k}(x)+\frac{\partial}{\partial x_{k}} B_{m, i}(x)=0,
$$

*Laboratoire de Mathématiques Paul Painlevé, CNRS UMR 8524/Université Lille 1 Sciences et Technologies, 59655 Villeneuve d'Ascq Cedex, France, alexandre.jollivet@math.univ-lille1.fr 
for $x \in \mathbb{R}^{n}$ and for $i, k, m=1 \ldots n$. For $\sigma \in(0,+\infty)$ we will denote by $\mathcal{B}(0, \sigma)$ (resp. $\overline{\mathcal{B}(0, \sigma)}$ ) the open (resp. closed) Euclidean ball of center 0 and radius $\sigma$.

When $n=3$ the equation (1.1) is the equation of motion of a particle of mass $m=1$ and charge $e=1$ in an external electromagnetic field described by $(V, B)$ (see, for example, [8, Section 17]). In this equation, $x, \dot{x}, \ddot{x}$ denote the position, the velocity and the acceleration of the particle respectively, and $t$ is the time.

We also assume throughout this paper that $F$ satisfies the following conditions

$$
F=F^{l}+F^{s},
$$

where $F^{l}(x, v):=-\nabla V^{l}(x)+B^{l}(x) v, F^{s}(x, v)=-\nabla V^{s}(x)+B^{s}(x) v$ and $\left(V^{l}, V^{s}\right) \in\left(C^{2}\left(\mathbb{R}^{n}, \mathbb{R}\right)\right)^{2},\left(B^{l}, B^{s}\right) \in\left(C^{1}\left(\mathbb{R}^{n}, A_{n}(\mathbb{R})\right)\right)^{2}$, and where

$$
\left|\partial_{x}^{j_{1}} V^{l}(x)\right| \leq \beta_{\left|j_{1}\right|}^{l}(1+|x|)^{-\left(\alpha+\left|j_{1}\right|\right)},\left|\partial_{x}^{j_{2}} B_{i, k}^{l}(x)\right| \leq \beta_{\left|j_{2}\right|+1}^{l}(1+|x|)^{-\left(\alpha+\left|j_{2}\right|+1\right)},
$$

$\left|\partial_{x}^{j_{1}} V^{s}(x)\right| \leq \beta_{\left|j_{1}\right|+1}^{s}(1+|x|)^{-\left(\alpha+1+\left|j_{1}\right|\right)},\left|\partial_{x}^{j_{2}} B_{i, k}^{s}(x)\right| \leq \beta_{\left|j_{2}\right|+2}^{s}(1+|x|)^{-\left(\alpha+\left|j_{2}\right|+2\right)}$,

for $x \in \mathbb{R}^{n},\left|j_{1}\right| \leq 2$ and $\left|j_{2}\right| \leq 1$ and for some $\alpha \in(0,1]$ (here $j$ is the multiindex $j=\left(j^{1}, \ldots, j^{n}\right) \in(\mathbb{N} \cup\{0\})^{n},|j|=\sum_{m=1}^{n} j^{m}$, and $\beta_{m}^{l}$ and $\beta_{m^{\prime}}^{s}$ are positive real constants for $m=0,1,2$ and for $\left.m^{\prime}=1,2,3\right)$. Although our electromagnetic fields are assumed to be smooth on the entire space, our study may provide interesting results even in presence of singularities.

For equation (1.1) we recall that the energy

$$
E=\frac{1}{2}|\dot{x}(t)|^{2}+V(x(t))
$$

is an integral of motion.

Set

$$
R_{0}=\frac{4 n \max \left(\beta_{1}^{l}, \beta_{2}^{l}\right)}{\alpha}, \mu^{l}=\frac{1}{\sqrt{n}}+2(1+\sqrt{n}) R_{0} .
$$

Then under conditions (1.4) the following is valid (see Section 4): for any $v \in \mathbb{R}^{n},|v| \geq \mu^{l}$, there exists a unique solution $z_{ \pm}(v,$.$) of the equation$

$$
\ddot{z}(t)=F^{l}(z(t), \dot{z}(t)),
$$

so that

$$
\dot{z}_{ \pm}(v, t)-v=o(1), \text { as } t \rightarrow \pm \infty, z_{ \pm}(v, 0)=0,
$$

and

$$
\sup _{\mathbb{R}}\left|z_{ \pm}(v, .)-v\right| \leq R_{0} \text { for } t \in \mathbb{R}
$$


When $F^{l} \equiv 0$ then we have $z_{ \pm}(v, t)=t v$ for $(t, v) \in \mathbb{R} \times \mathbb{R}^{n},|v|>\frac{1}{\sqrt{n}}$.

Then under conditions (1.4) and (1.5), the following is valid: for any $\left(v_{-}, x_{-}\right) \in \mathbb{R}^{n} \backslash \mathcal{B}\left(0, \mu^{l}\right) \times \mathbb{R}^{n}$, the equation (1.1) has a unique solution $x \in$ $C^{2}\left(\mathbb{R}, \mathbb{R}^{n}\right)$ such that

$$
x(t)=z_{-}\left(v_{-}, t\right)+x_{-}+y_{-}(t),
$$

where $\left|\dot{y}_{-}(t)\right|+\left|y_{-}(t)\right| \rightarrow 0$, as $t \rightarrow-\infty$; in addition for almost any $\left(v_{-}, x_{-}\right) \in$ $\mathbb{R}^{n} \backslash \mathcal{B}\left(0, \mu^{l}\right) \times \mathbb{R}^{n}$,

$$
x(t)=z_{+}\left(v_{+}, t\right)+x_{+}+y_{+}(t),
$$

for a unique $\left(v_{+}, x_{+}\right) \in \mathbb{R}^{n} \times \mathbb{R}^{n}$, where $\left|v_{+}\right|=\left|v_{-}\right| \geq \mu^{l}$ by conservation of the energy (1.6), and where $v_{+}=: a\left(v_{-}, x_{-}\right), x_{+}=: b\left(v_{-}, x_{-}\right)$, and $\left|\dot{y}_{+}(t)\right|+$ $\left|y_{+}(t)\right| \rightarrow 0$, as $t \rightarrow+\infty$. A solution $x$ of (1.1) that satisfies (1.11) and (1.12) for some $\left(v_{-}, x_{-}\right), v_{-} \neq 0$, is called a scattering solution.

We call the map $S:\left(\mathbb{R}^{n} \backslash \mathcal{B}\left(0, \mu^{l}\right)\right) \times \mathbb{R}^{n} \rightarrow\left(\mathbb{R}^{n} \backslash \mathcal{B}\left(0, \mu^{l}\right)\right) \times \mathbb{R}^{n}$ given by the formulas

$$
v_{+}=a\left(v_{-}, x_{-}\right), x_{+}=b\left(v_{-}, x_{-}\right),
$$

the scattering map for the equation (1.1). In addition, $a\left(v_{-}, x_{-}\right), b\left(v_{-}, x_{-}\right)$ are called the scattering data for the equation (1.1), and we define

$$
a_{s c}\left(v_{-}, x_{-}\right)=a\left(v_{-}, x_{-}\right)-v_{-}, \quad b_{s c}\left(v_{-}, x_{-}\right)=b\left(v_{-}, x_{-}\right)-x_{-} .
$$

Our definition of the scattering map is derived from constructions given in $[3,1]$. We refer the reader to $[13,3,14,9,1]$ and references therein for the forward classical scattering theory.

By $\mathcal{D}(S)$ we denote the set of definition of $S$. Under the conditions (1.4) and (1.5) the map $S: \mathcal{D}(S) \rightarrow\left(\mathbb{R}^{n} \backslash \mathcal{B}\left(0, \mu^{l}\right)\right) \times \mathbb{R}^{n}$ is continuous, and $\operatorname{Mes}\left(\left(\left(\mathbb{R}^{n} \backslash \mathcal{B}\left(0, \mu^{l}\right)\right) \times \mathbb{R}^{n}\right) \backslash \mathcal{D}(S)\right)=0$ for the Lebesgue measure on $\mathbb{R}^{n} \times \mathbb{R}^{n}$. In addition the map $S$ is uniquely determined by its restriction to $\mathcal{M}(S)=\mathcal{D}(S) \cap \mathcal{M}$ and by $F^{l}$, where $\mathcal{M}=\left\{\left(v_{-}, x_{-}\right) \in \mathbb{R}^{n} \times \mathbb{R}^{n} \mid v_{-} \neq\right.$ $\left.\left.0,<v_{-}, x_{-}\right\rangle=0\right\}$ and $\langle.,$.$\rangle denotes the canonical scalar product of the$ Euclidean space $\mathbb{R}^{n}$.

One can imagine the following experimental setting that allows to measure the scattering data without knowing the electromagnetic field $(V, B)$ inside a (a priori bounded) region of interest. First find an electromagnetic field $\left(V^{l}, B^{l}\right)$ that generates the same long range effects as $(V, B)$ does. Then compute the solutions $z_{ \pm}(v,$.$) of equation (1.8). Then for a fixed \left(v_{-}, x_{-}\right) \in$ $\left(\mathbb{R}^{n} \backslash \mathcal{B}\left(0, \mu^{l}\right)\right) \times \mathbb{R}^{n}$ send a particle far away from the region of interest with a trajectory asymptotic to $x_{-}+z_{-}\left(v_{-},.\right)$at large and negative times. When the particle escapes any bounded region of the space at finite time, then detect the particle and find $S\left(v_{-}, x_{-}\right)=\left(v_{+}, x_{+}\right)$so that the trajectory of 
the particle is asymptotic to $x_{+}+z_{+}\left(v_{+},.\right)$at large and positive times far away from the bounded region of interest.

In this paper we consider the following inverse scattering problem for equation (1.1):

Given $S$ and given the long range tail $F^{l}$ of the force $F$, find $F^{s}$.

The main results of the present work consist in estimates and asymptotics for the scattering data $\left(a_{s c}, b_{s c}\right)$ and scattering solutions for the equation (1.1) and in application of these asymptotics and estimates to the inverse scattering problem (1.15) at high energies. Our main results include, in particular, Theorem 1.1 given below that provides the high energies asymptotics of the scattering data.

Consider

$$
T \mathbb{S}^{n-1}:=\left\{(\theta, x) \in \mathbb{S}^{n-1} \times \mathbb{R}^{n} \mid<\theta, x>=0\right\},
$$

and for any $m \in \mathbb{N}$ consider the x-ray transform $P$ defined by

$$
\operatorname{Pf}(\theta, x):=\int_{-\infty}^{+\infty} f(t \theta+x) d t
$$

for any function $f \in C\left(\mathbb{R}^{n}, \mathbb{R}^{m}\right)$ so that $|f(x)|=O\left(|x|^{-\tilde{\beta}}\right)$ as $|x| \rightarrow+\infty$ for some $\tilde{\beta}>1$.

Set

$$
\begin{aligned}
& W^{l}(v, x):=-\int_{-\infty}^{0} \int_{-\infty}^{\sigma}\left(\nabla V^{l}\left(z_{-}(v, \tau)+x\right)-\nabla V^{l}\left(z_{-}(v, \tau)\right)\right) d \tau d \sigma \\
& +\int_{-\infty}^{0} \int_{-\infty}^{\sigma}\left(B^{l}\left(z_{-}(v, \tau)+x+\int_{-\infty}^{\tau} \int_{-\infty}^{s_{1}}\left(B^{l}\left(z_{-}\left(v, s_{2}\right)+x\right)-B^{l}\left(z_{-}\left(v, s_{2}\right)\right)\right) \dot{z}_{-}\left(s_{2}\right) d s_{2} d s_{1}\right)\right. \\
& \left.-B^{l}\left(z_{-}(v, \tau)\right)\right) \dot{z}_{-}(v, \tau) d \tau d \sigma \\
& +\int_{-\infty}^{0} \int_{-\infty}^{\sigma} B^{l}\left(z_{-}(v, \tau)+x\right)\left(\int_{-\infty}^{\tau}\left(B^{l}\left(z_{-}(v, \eta)+x\right)-B^{l}\left(z_{-}(v, \eta)\right)\right) \dot{z}_{-}(v, \eta) d \eta\right) d \tau d \sigma \\
& +\int_{0}^{+\infty} \int_{\sigma}^{+\infty}\left(\nabla V^{l}\left(z_{+}(a, \tau)+b\right)-\nabla V^{l}\left(z_{+}(a, \tau)\right)\right) d \tau d \sigma \\
& +\int_{0}^{+\infty} \int_{\sigma}^{+\infty} B^{l}\left(z_{+}(a, \tau)+b\right)\left(\int_{\tau}^{+\infty}\left(B^{l}\left(z_{+}(a, \eta)+b\right)-B^{l}\left(z_{+}(a, \eta)\right)\right) \dot{z}_{+}(a, \eta) d \eta\right) d \tau d \sigma \\
& -\int_{0}^{+\infty} \int_{\sigma}^{+\infty}\left(B^{l}\left(z_{+}(a, \tau)+b+\int_{\tau}^{+\infty} \int_{s_{1}}^{+\infty}\left(B^{l}\left(z_{+}\left(a, s_{2}\right)+b\right)-B^{l}\left(z_{+}\left(a, s_{2}\right)\right)\right) \dot{z}_{+}\left(a, s_{2}\right) d s_{2} d s_{1}\right)\right. \\
& \left.-B^{l}\left(z_{+}(a, \tau)\right)\right) \dot{z}_{+}(a, \tau) d \tau d \sigma,
\end{aligned}
$$


for $(v, x) \in \mathcal{D}(S)$, In (1.16), $a(v, x)$ and $b(v, x)$ are shortened to $a, b$. The vector valued map $W^{l}$ is known from $F^{l}$ and from the scattering data. Then we have the following asymptotic results.

Theorem 1.1. Let $(\theta, x) \in T \mathbb{S}^{n-1}$. Under conditions (1.4) and (1.5) the following limits are valid $a_{s c}(\rho \theta, x)=\int_{-\infty}^{+\infty} B(\tau \theta+x) \theta d \tau+\rho^{-1}\left(-P(\nabla V)(\theta, x)+W_{1}\left(B^{l}, B^{s}, \theta, x\right)\right)+o\left(\rho^{-1}\right)$,

as $\rho \rightarrow+\infty$, and

$$
\begin{aligned}
b_{s c}(\rho \theta, x)= & W^{l}(\rho \theta, x)+\rho^{-1}\left(\int_{-\infty}^{0} \int_{-\infty}^{\sigma} B^{s}(\tau \theta+x) \theta d \tau d \sigma-\int_{0}^{+\infty} \int_{\sigma}^{+\infty} B^{s}(\tau \theta+x) \theta d \tau d \sigma\right) \\
& +\rho^{-2}\left(\int_{-\infty}^{0} \int_{-\infty}^{\sigma}\left(-\nabla V^{s}\right)(\tau \theta+x) d \tau d \sigma-\int_{0}^{+\infty} \int_{\sigma}^{+\infty}\left(-\nabla V^{s}\right)(\tau \theta+x) d \tau d \sigma\right. \\
& \left.+W_{2}\left(B^{l}, B^{s}, \theta, x\right)\right)+o\left(\rho^{-2}\right),
\end{aligned}
$$

as $\rho \rightarrow+\infty$, where

$$
\begin{aligned}
W_{1}\left(B^{l}, B^{s}, \theta, x\right)= & \int_{\mathbb{R}} B(\tau \theta+x)\left(\int_{-\infty}^{\tau} B(\sigma \theta+x) \theta d \sigma\right) d \tau \\
& +\sum_{k=1}^{n} \theta_{k} \int_{\mathbb{R}}\left(<\nabla B_{j, k}(\tau \theta+x), \int_{-\infty}^{\tau} \int_{-\infty}^{\sigma}\left(B(\eta \theta+x)-B^{l}(\eta \theta)\right) \theta d \eta d \sigma\right. \\
& \left.+\int_{0}^{\tau} \int_{-\infty}^{\sigma} B^{l}(\eta \theta) \theta d \eta d \sigma>\right)_{j=1 \ldots n} d \tau
\end{aligned}
$$

and

$$
\begin{aligned}
& W_{2}\left(B^{l}, B^{s}, \theta, x\right)=\int_{-\infty}^{0} \int_{-\infty}^{\sigma} B^{s}(\tau \theta+x)\left(\int_{-\infty}^{\tau} B(\eta \theta+x) \theta d \eta\right) d \tau d \sigma \\
& -\int_{0}^{+\infty} \int_{\sigma}^{+\infty} B^{s}(\tau \theta+x)\left(\int_{-\infty}^{\tau} B(\eta \theta+x) \theta d \eta\right) d \tau d \sigma \\
& +\int_{-\infty}^{0} \int_{-\infty}^{\sigma} B^{l}(\tau \theta+x)\left(\int_{-\infty}^{\tau} B^{s}(\eta \theta+x) \theta d \eta\right) d \tau d \sigma \\
& +\int_{0}^{+\infty} \int_{\sigma}^{+\infty} B^{l}(\tau \theta+x)\left(\int_{\tau}^{+\infty} B^{s}(\eta \theta+x) \theta d \eta\right) d \tau d \sigma \\
& +\sum_{k=1}^{n} \theta_{k} \int_{-\infty}^{0} \int_{-\infty}^{\sigma}\left(<\nabla B_{j, k}^{s}(\tau \theta+x), \int_{-\infty}^{\tau} \int_{-\infty}^{\eta_{1}}\left(B\left(\eta_{2} \theta+x\right)-B^{l}\left(\eta_{2} \theta\right)\right) \theta d \eta_{2} d \eta_{1}\right.
\end{aligned}
$$




$$
\begin{aligned}
& \left.+\int_{0}^{\tau} \int_{-\infty}^{\eta_{1}} B^{l}\left(\eta_{2} \theta\right) \theta d \eta_{2} d \eta_{1}>\right)_{j=1 \ldots n} d \tau d \sigma \\
& +\sum_{k=1}^{n} \theta_{k} \int_{-\infty}^{0} \int_{-\infty}^{\sigma}\left(<\nabla B_{j, k}^{l}(\tau \theta+x), \int_{-\infty}^{\tau} \int_{-\infty}^{\eta_{1}} B^{s}\left(\eta_{2} \theta+x\right) \theta d \eta_{2} d \eta_{1}>\right)_{j=1 \ldots n} d \tau d \sigma \\
& -\sum_{k=1}^{n} \theta_{k} \int_{0}^{+\infty} \int_{\sigma}^{+\infty}\left(<\nabla B_{j, k}^{s}(\tau \theta+x), \int_{-\infty}^{\tau} \int_{-\infty}^{\eta_{1}}\left(B\left(\eta_{2} \theta+x\right)-B^{l}\left(\eta_{2} \theta\right)\right) \theta d \eta_{2} d \eta_{1}\right. \\
& \left.+\int_{0}^{\tau} \int_{-\infty}^{\eta_{1}} B^{l}\left(\eta_{2} \theta\right) \theta d \eta_{2} d \eta_{1}>\right)_{j=1 \ldots n} d \tau d \sigma \\
& -\sum_{k=1}^{n} \theta_{k} \int_{0}^{+\infty} \int_{\sigma}^{+\infty}\left(<\nabla B_{j, k}^{l}(\tau \theta+x), \int_{\tau}^{+\infty} \int_{\eta_{1}}^{+\infty} B^{s}\left(\eta_{2} \theta+x\right) \theta d \eta_{2} d \eta_{1}>\right)_{j=1 \ldots n} d \tau d \sigma .
\end{aligned}
$$

From (1.17) and [4, Proposition 1.2] and inversion of the x-ray transform (see $[12,2,10,11]$ ) it follows that $F^{s}$ can be reconstructed from the high energies asymptotics of $a_{s c}$ and $F^{l}$. From (1.18) one can prove the following statements (see [4, Proposition 1.2] and [7]): The magnetic field $B^{s}$ can be reconstructed from $W^{l}, B^{l}$ and the high energies asymptotics of $b_{s c}$ when $n \geq 3$, and up to its radial part when $n=2$; The potential $V^{s}$ is uniquely determined up to its radial part by $W^{l}, B^{l}$ and the high energies asymptotics of $b_{s c}$ when $n \geq 3$; The potential $V^{s}$ is not uniquely determined up to its radial part by $W^{l}, B^{l}$ and the high energies asymptotics of $b_{s c}$ given above when $n=2$.

Theorem 1.1 is a generalization of [4, formulas (1.10)-(1.13)] where inverse scattering for the multidimensional Newton equation was studied in the short range case $\left(F^{l} \equiv 0\right)$.

Inverse scattering at high energies for the multidimensional Newton equation in a short range potential $V$ was first studied by [11]. Then inverse scattering at high energies for this latter equation in a long range potential $V$ was studied by [6]. We develop the approach of $[11,6]$ to obtain the results of the present work.

A similar study was done for the multidimensional relativistic Newton equation in a long range electromagnetic field [7]. However in our opinion there is an interesting difference between the high energies asymptotics of the scattering data of the nonrelativistic and relativistic Newton equations. Indeed the high energies asymptotics of the velocity component $a^{r}$ of the scattering map for the relativistic Newton equation is [7, Theorem 1.1]:

$$
\frac{\rho}{\sqrt{1-\frac{\rho^{2}}{c^{2}}}}\left(a^{r}(\rho \theta, x)-\rho \theta\right)=-P(\nabla V)(\theta, x)+\int_{-\infty}^{+\infty} B(\tau \theta+x) \theta d \tau+o\left(\sqrt{1-\frac{\rho}{c}}\right),
$$


as $\rho \rightarrow c, \rho<c$, and for any $(\theta, x) \in T \mathbb{S}^{n-1}$, where $c>0$ denotes the speed of light. Hence the leading term of the above asymptotics depends on both magnetic and electric fields $B$ and $-\nabla V$, whereas in the nonrelativistic case the leading high energies term of $a_{s c}$ depends on the magnetic field $B$ only (and is independent of the electric one). This difference is quite interesting in our opinion.

For inverse scattering at fixed energy for the multidimensional Newton equation, see for example [5] and references therein. For the inverse scattering problem in quantum mechanics, see references given in [4].

Our paper is organized as follows. In Section 2 we transform the differential equation (1.1) with initial conditions (1.11) into an integral equation which takes the form $y_{-}=A\left(y_{-}\right)$. Then we study the operator $A$ on a suitable space (Lemma 2.1) and we give estimates for the deflection $y_{-}(t)$ in (1.11) and for the scattering data $a_{s c}\left(v_{-}, x_{-}\right), b_{s c}\left(v_{-}, x_{-}\right)$(Theorem 2.4). We provide the Born approximation of the scattering data at fixed energy $E=\frac{1}{2}$. We will always work with small angle scattering compared to the dynamics generated by $F^{l}$ through the "free" solutions $z_{-}\left(v_{-}, t\right)$ : In particular, the angle between the vectors $\dot{x}(t)=\dot{z}_{-}\left(v_{-}, t\right)+\dot{y}_{-}(t)$ and $\dot{z}_{-}\left(v_{-}, t\right)$ goes to zero when the parameters $\beta:=\max \left(\beta_{1}^{l}, \beta_{2}^{l}, \beta_{2}^{s}, \beta_{3}^{s}\right), \alpha, n, v_{-} /\left|v_{-}\right|, x_{-}$are fixed and $\left|v_{-}\right|$increases. In Section 3 we change the definition of the scattering map so that one can obtain for the modified scattering data $\left(\tilde{a}_{s c}\left(v_{-}, x_{-}\right), \tilde{b}_{s c}\left(v_{-}, x_{-}\right)\right)$ their approximation at high energies, or their Born approximation at fixed energy, or their approximation when the parameters $\alpha, n, v_{-}$and $\beta$ are fixed and $\left|x_{-}\right| \rightarrow+\infty$. This latter asymptotic regime is not covered by Theorems 1.1 and 2.4. Sections 4, 5 and 6 are devoted to proofs of our Theorems and Lemmas.

\section{Scattering solutions}

\subsection{Integral equation}

For the rest of the text we set

$$
\beta_{2}:=\max \left(\beta_{2}^{l}, \beta_{2}^{s}\right), \beta:=\max \left(\beta_{1}^{l}, \beta_{2}, \beta_{3}^{s}\right) .
$$

For the rest of the text $H(f(\tau), \dot{f}(\tau))$ is shortened to $H(f)(\tau)$ for any $(f, \tau) \in C^{1}\left(\mathbb{R}, \mathbb{R}^{n}\right) \times \mathbb{R}$, where $H$ stands for $F, F^{s}$ or $F^{l}$.

Let $\left(v_{-}, x_{-}\right) \in \mathbb{R}^{n} \times \mathbb{R}^{n},\left\langle v_{-}, x_{-}>=0\right.$ and $\left|v_{-}\right| \geq \mu^{l}$, where $\mu^{l}$ is defined in (1.7). Then the function $y_{-}$in (1.11) satisfies the integral equation $y_{-}=A\left(y_{-}\right)$where

$$
A(f)(t)=\int_{-\infty}^{t} \dot{A}(f)(\sigma) d \sigma
$$




$$
\dot{A}(f)(t)=\int_{-\infty}^{t}\left(F\left(z_{-}\left(v_{-}, .\right)+x_{-}+f\right)(\tau)-F^{l}\left(z_{-}\left(v_{-}, .\right)\right)(\tau)\right) d \tau
$$

for $t \in \mathbb{R}$ and for $f \in C^{1}\left(\mathbb{R}, \mathbb{R}^{n}\right), \sup _{(-\infty, 0]}(|f|+|\dot{f}|)<\infty$. We have $A(f) \in$ $C^{2}\left(\mathbb{R}, \mathbb{R}^{n}\right)$ for $f \in C^{1}\left(\mathbb{R}, \mathbb{R}^{n}\right)$ so that $\sup _{(-\infty, 0]}(|f|+|\dot{f}|)<\infty$ (see (4.2) and $(4.3))$.

Let $R \in(0,+\infty)$ we set

$$
R^{\prime}=R_{0}+R
$$

where $R_{0}$ is defined in (1.7). Let $r \in(0,1)$. For $\left|v_{-}\right| \geq \mu^{l},\left|v_{-}\right| \geq \sqrt{2} R^{\prime}$, we introduce the following complete metric space $M_{R, r}$ endowed with the following norm $\|\cdot\|$

$$
\begin{gathered}
M_{R, r}=\left\{f \in C^{1}\left(\mathbb{R}, \mathbb{R}^{n}\right) \mid\|f\| \leq R\right\} \\
\|f\|=\max \left(\sup _{t \in(-\infty, 0)} \max \left(1,\left(1-r+\left(\frac{\left|v_{-}\right|}{\sqrt{2}}-R^{\prime}\right)|t|\right)\right)|\dot{f}(t)|, \sup _{(0,+\infty)}|\dot{f}|\right. \\
\left.\left(\frac{\left|v_{-}\right|}{\sqrt{2}}-R^{\prime}\right) \sup _{(-\infty, 0)}|f|\right) .
\end{gathered}
$$

The space $M_{R, r}$ is a convex subset of $C^{1}\left(\mathbb{R}, \mathbb{R}^{n}\right)$. Then we have the following estimate and contraction estimate for the map $A$ restricted to $M_{R, r}$.

Lemma 2.1. Let $(R, r) \in(0,+\infty) \times(0,1)$. Let $\left(v_{-}, x_{-}\right) \in\left(\mathbb{R}^{n} \backslash \mathcal{B}\left(0, \mu^{l}\right)\right) \times \mathbb{R}^{n}$, $<v_{-}, x_{-}>=0,\left|v_{-}\right| \geq \max \left(\mu^{l}, \sqrt{2} R_{0}+\sqrt{2} R\left(1+r^{-1}\right)\right)$. Then the following estimates are valid

$$
\begin{aligned}
\|A(f)\| & \leq \lambda_{1}\left(n, \alpha, \beta_{1}^{l}, \beta_{2},\left|x_{-}\right|,\left|v_{-}\right|, r\right) \\
& :=2 n \frac{\beta_{1}^{l} R+\beta_{2}\left(\left|x_{-}\right|+2\right)\left(1+\sqrt{n}\left(\left|v_{-}\right|+R^{\prime}\right)\right)}{\alpha\left(\frac{\left|v_{-}\right|}{\sqrt{2}}-R^{\prime}\right)(1-r)^{\alpha+1}},
\end{aligned}
$$

and

$$
\begin{gathered}
\left\|A\left(f_{1}\right)-A\left(f_{2}\right)\right\| \leq \lambda_{2}\left(n, \alpha, \beta_{2}, \beta_{3}^{s},\left|v_{-}\right|, r\right)\left\|f_{1}-f_{2}\right\|, \\
\lambda_{2}:=\frac{2 n \beta\left(1+\frac{1}{1-r}\right)^{2}}{\alpha\left(\frac{\left|v_{-}\right|}{\sqrt{2}}-R^{\prime}\right)(1-r)^{\alpha}}\left(1+\frac{1+\sqrt{n}\left(\left|v_{-}\right|+R^{\prime}\right)}{\frac{\left|v_{-}\right|}{\sqrt{2}}-R^{\prime}}\right),
\end{gathered}
$$

for $\left(f, f_{1}, f_{2}\right) \in M_{R, r}^{3}$.

A proof of Lemma 2.1 is given in Section 5 .

We also need the following result. 
Lemma 2.2. Let $(R, r) \in(0,+\infty) \times(0,1)$. Let $\left(v_{-}, x_{-}\right) \in\left(\mathbb{R}^{n} \backslash \mathcal{B}\left(0, \mu^{l}\right)\right) \times \mathbb{R}^{n}$, $<v_{-}, x_{-}>=0,\left|v_{-}\right| \geq \max \left(\mu^{l}, \sqrt{2} R_{0}+\sqrt{2} R\left(1+r^{-1}\right)\right)$. When $y_{-} \in M_{R, r}$ is a fixed point for the map $A$ then $x:=z_{-}\left(v_{-},.\right)+x_{-}+y_{-}$is a scattering solution for equation (1.1) and

$$
x(t)=z_{+}\left(a\left(v_{-}, x_{-}\right), t\right)+b\left(v_{-}, x_{-}\right)+y_{+}(t),
$$

for $t \geq 0$, where

$$
\begin{gathered}
a\left(v_{-}, x_{-}\right):=v_{-}+\int_{-\infty}^{+\infty} F(x)(\tau) d \tau, \\
b\left(v_{-}, x_{-}\right):=x_{-}+y_{-}(0)-y_{+}(0), \\
y_{+}(t):=\int_{t}^{+\infty} \int_{\sigma}^{+\infty}\left(F(x)-F^{l}\left(z_{+}\left(a\left(v_{-}, x_{-}\right)\right)\right)(\tau) d \tau d \sigma,\right.
\end{gathered}
$$

for $t \geq 0$.

Lemma 2.2 is proved in Section 4.

Note that

$$
\frac{1+\sqrt{n}\left(\left|v_{-}\right|+R^{\prime}\right)}{\frac{\left|v_{-}\right|}{\sqrt{2}}-R^{\prime}} \leq 2 \sqrt{2 n}
$$

when $\left|v_{-}\right| \geq \frac{1}{\sqrt{n}}+(1+2 \sqrt{2}) R^{\prime}$. Then we obtain the following Corollary of Lemma 2.1.

Corollary 2.3. Let $x_{-} \in \mathbb{R}^{n}$ and let $r \in(0,1)$. Set

$$
R:=\frac{2^{\frac{9}{2}} n^{\frac{3}{2}} \beta\left(\left|x_{-}\right|+2\right)}{\alpha(1-r)^{\alpha+1}}
$$

and

$$
\rho\left(\alpha, \beta, r,\left|x_{-}\right|\right)=\mu^{l}+\left(1+\sqrt{2}\left(1+r^{-1}\right)\right) R+\frac{16 \sqrt{2} n \beta(1+2 \sqrt{2 n})}{\alpha(1-r)^{\alpha+2}} .
$$

Then for any $v_{-} \in \mathbb{R}^{n}$ so that

$$
\left|v_{-}\right| \geq \rho\left(\alpha, \beta, r,\left|x_{-}\right|\right)
$$

the map A defined by (2.2) and (2.3) is a $\frac{1}{2}$-contraction from $M_{R, r}$ to $M_{R, r}$. 


\subsection{Estimates on the scattering solutions}

In this Section our main results consist in estimates and asymptotics for the scattering data $\left(a_{s c}, b_{s c}\right)$ and scattering solutions for the equation (1.1). Let $\left(v_{-}, x_{-}\right) \in \mathcal{D}(S)$. Set

$$
\begin{aligned}
\Delta\left(v_{-}, x_{-}\right):= & a_{s c}\left(v_{-}, x_{-}\right)+\int_{-\infty}^{+\infty} \nabla V\left(\tau v_{-}+x_{-}\right) d \tau \\
& -\int_{-\infty}^{+\infty} B\left(\tau v_{-}+x_{-}\right)\left(v_{-}+\int_{-\infty}^{\tau} B\left(\sigma v_{-}+x_{-}\right) v_{-} d \sigma\right) d \tau-W\left(v_{-}, x_{-}\right),
\end{aligned}
$$

where

$$
\begin{aligned}
& W\left(v_{-}, x_{-}\right):=\int_{-\infty}^{+\infty}\left(B \left(z_{-}(\tau)+x_{-}\right.\right. \\
& \left.\left.+\int_{-\infty}^{\tau} \int_{-\infty}^{s_{1}}\left(B\left(z_{-}\left(s_{2}\right)+x_{-}\right)-B^{l}\left(z_{-}\left(s_{2}\right)\right)\right) \dot{z}_{-}\left(s_{2}\right) d s_{2} d s_{1}\right)-B\left(\tau v_{-}+x_{-}\right)\right) v_{-} d \tau
\end{aligned}
$$

and set

$$
\begin{aligned}
& \Omega\left(v_{-}, x_{-}\right):=b_{s c}\left(v_{-}, x_{-}\right)-W^{l}\left(v_{-}, x_{-}\right)-\int_{-\infty}^{0} \int_{-\infty}^{\sigma} F^{s}\left(. v_{-}+x_{-}\right)(\tau) d \tau d \sigma \\
& +\int_{0}^{+\infty} \int_{\sigma}^{+\infty} F^{s}\left(. v_{-}+x_{-}\right)(\tau) d \tau d \sigma-\int_{-\infty}^{0} \int_{-\infty}^{\sigma} B^{s}\left(\tau v_{-}+x_{-}\right) \int_{-\infty}^{\tau} B\left(s_{1} v_{-}+x_{-}\right) v_{-} d s_{1} d \tau d \sigma \\
& +\int_{0}^{+\infty} \int_{\sigma}^{+\infty} B^{s}\left(\tau v_{-}+x_{-}\right)\left(\int_{-\infty}^{\tau} B\left(s_{1} v_{-}+x_{-}\right) v_{-} d s_{1}\right) d \tau d \sigma \\
& -W^{l, s}\left(v_{-}, x_{-}\right)
\end{aligned}
$$

where $W^{l}$ is defined in (1.16) and

$$
\begin{gathered}
W^{l, s}\left(v_{-}, x_{-}\right):=\int_{-\infty}^{0} \int_{-\infty}^{\sigma}\left(B ^ { s } \left(z_{-}(\tau)+x_{-}\right.\right. \\
\left.\left.+\int_{-\infty}^{\tau} \int_{-\infty}^{s_{1}}\left(B\left(z_{-}\left(s_{2}\right)+x_{-}\right)-B^{l}\left(z_{-}\left(s_{2}\right)\right)\right) \dot{z}_{-}\left(s_{2}\right) d s_{2} d s_{1}\right)-B^{s}\left(\tau v_{-}+x_{-}\right)\right) v_{-} d \tau d \sigma \\
+\int_{-\infty}^{0} \int_{-\infty}^{\sigma}\left(B^{l}\left(z_{-}(\tau)+x_{-}+\int_{-\infty}^{\tau} \int_{-\infty}^{s_{1}}\left(B\left(z_{-}\left(s_{2}\right)+x_{-}\right)-B^{l}\left(z_{-}\left(s_{2}\right)\right)\right) \dot{z}_{-}\left(s_{2}\right) d s_{2} d s_{1}\right)\right. \\
\left.-B^{l}\left(z_{-}(\tau)+x_{-}+\int_{-\infty}^{\tau} \int_{-\infty}^{s_{1}}\left(B^{l}\left(z_{-}\left(s_{2}\right)+x_{-}\right)-B^{l}\left(z_{-}\left(s_{2}\right)\right)\right) \dot{z}_{-}\left(s_{2}\right) d s_{2} d s_{1}\right)\right) \dot{z}_{-}(\tau) d \tau d \sigma \\
+\int_{-\infty}^{0} \int_{-\infty}^{\sigma} B^{l}\left(z_{-}(\tau)+x_{-}\right)\left(\int_{-\infty}^{\tau} B^{s}\left(z_{-}\left(s_{1}\right)+x_{-}\right) \dot{z}_{-}\left(s_{1}\right) d s_{1}\right) d \tau d \sigma
\end{gathered}
$$




$$
\begin{aligned}
& -\int_{0}^{+\infty} \int_{\sigma}^{+\infty} B^{s}\left(z_{-}(\tau)+x_{-}+\int_{-\infty}^{\tau} \int_{-\infty}^{s_{1}}\left(B\left(z_{-}\left(s_{2}\right)+x_{-}\right)-B^{l}\left(z_{-}\left(s_{2}\right)\right)\right) \dot{z}_{-}\left(s_{2}\right) d s_{2} d s_{1}\right) \\
& \left.-B^{s}\left(\tau v_{-}+x_{-}\right)\right) v_{-} d \tau d \sigma-\int_{0}^{+\infty} \int_{\sigma}^{+\infty}\left(B ^ { l } \left(z_{+}(a, \tau)+x_{+}\right.\right. \\
& \left.+\int_{\tau}^{+\infty} \int_{s_{1}}^{+\infty}\left(B\left(z_{+}\left(a, s_{2}\right)+x_{+}\right)-B^{l}\left(z_{+}\left(a, s_{2}\right)\right)\right) \dot{z}_{+}\left(a, s_{2}\right) d s_{2} d s_{1}\right)-B^{l}\left(z_{+}(a, \tau)+x_{+}\right. \\
& \left.\left.+\int_{\tau}^{+\infty} \int_{s_{1}}^{+\infty}\left(B^{l}\left(z_{+}\left(a, s_{2}\right)+x_{+}\right)-B^{l}\left(z_{+}\left(a, s_{2}\right)\right)\right) \dot{z}_{+}\left(a, s_{2}\right) d s_{2} d s_{1}\right)\right) \dot{z}_{+}(a, \tau) d \tau d \sigma \\
& +\int_{0}^{+\infty} \int_{\sigma}^{+\infty} B^{l}\left(z_{+}(a, \tau)+x_{+}\right)\left(\int_{\tau}^{+\infty} B^{s}\left(z_{+}(a, \eta)+x_{+}\right) \dot{z}_{+}(a, \eta) d \eta\right) d \tau d \sigma .
\end{aligned}
$$

(In (2.18) and (2.20) we shorten $z_{-}\left(v_{-},.\right)$and $a\left(v_{-}, x_{-}\right)$to $z_{-}$and $a$.) Vectors $W$ and $W^{l, s}$ depend on $S, F^{l}$ and $B^{s}$. Then we have the following result.

Theorem 2.4. Let $\left(v_{-}, x_{-}, r\right) \in \mathbb{R}^{n} \times \mathbb{R}^{n} \times(0,1)$ so that $\left\langle v_{-}, x_{-}>=0\right.$ and

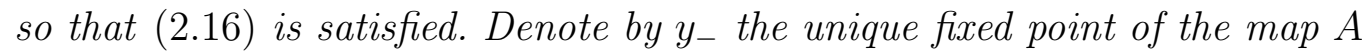
in $M_{R, r}$ where $R$ is defined by (2.14). Then the following estimates are valid:

$$
\left|\dot{y}_{-}(t)\right| \leq \frac{2^{\frac{5}{2}} n^{\frac{3}{2}} \beta_{2}\left(\left|x_{-}\right|+2\right)}{(\alpha+1)\left(1-r-\left(\frac{\left|v_{-}\right|}{\sqrt{2}}-R^{\prime}\right) t\right)^{\alpha+1}},
$$

for $t \leq 0$,

$$
\sup _{(0,+\infty)}\left|\dot{y}_{-}\right| \leq \frac{2^{\frac{7}{2}} n^{\frac{3}{2}} \beta_{2}\left(\left|x_{-}\right|+2\right)}{(\alpha+1)(1-r)^{\alpha+1}} .
$$

In addition

$$
\begin{gathered}
\left|a_{s c}\left(v_{-}, x_{-}\right)\right| \leq \frac{2^{\frac{5}{2}} n}{\left(1+\frac{\left|x_{-}\right|}{\sqrt{2}}-r\right)^{\alpha}}\left(\frac{\beta_{1}^{l}}{\alpha}+\frac{\beta_{2}}{(\alpha+1)\left(1+\frac{\left|x_{-}\right|}{\sqrt{2}}-r\right)}\right) \\
\left|b_{s c}\left(v_{-}, x_{-}\right)\right| \leq \frac{2^{\frac{7}{2}} n^{\frac{3}{2}} \beta_{2}\left(\left|x_{-}\right|+2\right)}{\alpha(\alpha+1)\left(\frac{\left|v_{-}\right|}{\sqrt{2}}-R^{\prime}\right)(1-r)^{\alpha}} \\
\left|\dot{y}_{+}(t)\right| \leq \frac{2^{\frac{5}{2}} n^{\frac{3}{2}} \beta_{2}\left(\left|x_{-}\right|+2\right)}{(\alpha+1)\left(1-r+t\left(\frac{\left|v_{-}\right|}{\sqrt{2}}-R^{\prime}\right)\right)^{\alpha+1}}
\end{gathered}
$$

for $t \geq 0$, and

$$
\begin{gathered}
\left|\Delta\left(v_{-}, x_{-}\right)\right| \leq \frac{600 n^{3} \beta^{2}\left(1+R^{\prime}+\left|x_{-}\right|\right)}{\alpha^{2}\left(\frac{\left|v_{-}\right|}{\sqrt{2}}-R^{\prime}\right)^{2}(1-r)^{2 \alpha+3}}, \\
\left|\Omega\left(v_{-}, x_{-}\right)\right| \leq \frac{700 n^{3} \beta^{2}\left(1+R^{\prime}\right)\left(1+\left|x_{-}\right|\right)}{\alpha^{2}\left(\frac{\left|v_{-}\right|}{\sqrt{2}}-R^{\prime}\right)^{3}(1-r)^{2 \alpha+2}} .
\end{gathered}
$$

Theorem 2.4 is proved in Section 6 . 


\subsection{Born approximation at fixed energy $E=\frac{1}{2}$}

Estimates (2.26) and (2.27) also provide the asymptotics of the scattering data $\left(a_{s c}(v, x), b_{s c}(v, x)\right)$ when $v \in \mathbb{S}^{n-1}, x, n$ and $\alpha$ are fixed and $\beta$ goes to 0 , i.e. they provide the Born approximation of the scattering data at fixed energy $E=\frac{1}{2}$. In more details, we have

$$
a_{s c}(\theta, x)=-P(\nabla V)(\theta, x) d \tau+\int_{-\infty}^{+\infty} B(\tau \theta+x) \theta d \tau+O\left(\beta^{2}\right),
$$

and

$$
\begin{aligned}
b_{s c}(\theta, x)= & W^{l}(\theta, x)+\int_{-\infty}^{0} \int_{-\infty}^{\sigma} B^{s}(\tau \theta+x) \theta d \tau d \sigma \\
& -\int_{0}^{+\infty} \int_{\sigma}^{+\infty} B^{s}(\tau \theta+x) \theta d \tau d \sigma-\int_{-\infty}^{0} \int_{-\infty}^{\sigma} \nabla V^{s}(\tau \theta+x) d \tau d \sigma \\
& +\int_{0}^{+\infty} \int_{\sigma}^{+\infty} \nabla V^{s}(\tau \theta+x) d \tau d \sigma+O\left(\beta^{2}\right),
\end{aligned}
$$

for $(\theta, x) \in T \mathbb{S}^{n-1}$, as $\beta \rightarrow 0^{+}$. Then for the recovery of the force field from the Born approximation at fixed energy of the scattering data we have (see [7]): one can reconstruct the force field $(\nabla V, B)$ from the Born approximation of $a_{s c}$ at fixed energy; The Born approximation of $b_{s c}$ at fixed energy and $W^{l}$ determine $V^{s}$ up to spherical symmetric potentials, and they uniquely determine $B^{s}$ when $n \geq 3$, and they uniquely determine $B^{s}$ up to magnetic fields that are spherical symmetric in each of its components when $n=2$.

\section{$3 \quad$ Further comments}

For a solution $x$ at a nonzero energy for equation (1.1) we say that it is a scattering solution when there exists $\varepsilon>0$ so that $1+|x(t)| \geq \varepsilon(1+|t|)$ for $t \in \mathbb{R}$ (see [1]). In the Introduction and in the previous Section we choose to parametrize the scattering solutions of equation (1.1) by the solutions $z_{ \pm}(v,$.$) of the equation (1.8) (see the asymptotic behaviors (1.11) and$ $(1.12)$ ), and then to formulate the inverse scattering problem (1.15) using this parametrization. We obtain the estimates (2.26) and (2.27) from which are derived the high energies asymptotics and the Born approximation at fixed energy of the scattering data. However these estimates do not provide the asymptotics of the scattering data $\left(a_{s c}(v, x), b_{s c}(v, x)\right)$ when the parameters $\alpha, n, v$ and $\beta$ are fixed and $|x| \rightarrow+\infty$. Motivated by this disadvantage, we introduce below a new family of "free" solutions $z_{ \pm}(v, x,$.$) of equation (1.8)$ 
that will be used for parametrizing some unbounded solutions of the Newton equation (1.1) at nonzero energy and for measuring their deviation.

We set

$$
R_{0}(\sigma):=\frac{16 \max \left(\beta_{1}^{l}, \beta_{2}^{l}\right) n}{\alpha\left(\frac{1}{2}+\frac{\sigma}{\sqrt{2}}\right)^{\alpha}}, \mu^{l}(\sigma):=\frac{1}{\sqrt{n}}+10 R_{0}(\sigma),
$$

for $\sigma \geq 0$. Let $(v, x) \in \mathbb{R}^{n} \times \mathbb{R}^{n}$ so that $\left\langle v, x>=0\right.$ and $|v| \geq \mu^{l}(|x|)$. Then for any $(w, q) \in \mathcal{B}\left(v, \frac{|v|}{2^{\frac{5}{2}}}\right) \times \overline{\mathcal{B}\left(0, \frac{1}{2}\right)},|w|=|v|$, there exists a unique solution $z_{ \pm}(w, x+q,$.$) of the equation (1.8) so that$

$$
\dot{z}_{ \pm}(w, x+q, t)-w=o(1) \text { as } t \rightarrow \pm \infty, z_{ \pm}(w, x+q, 0)=x+q,
$$

and

$$
\sup _{\mathbb{R}}\left|\dot{z}_{ \pm}(w, x+q, .)-w\right| \leq R_{0}(|x|) .
$$

We will now define our modified scattering data. For $\left(v_{-}, x_{-}\right) \in \mathbb{R}^{n} \times \mathbb{R}^{n}$, $\left.<v_{-}, x_{-}\right\rangle=0$, so that $\left|v_{-}\right| \geq \mu_{l}(|x|)$, then there exists a unique solution $x \in C^{2}\left(\mathbb{R}, \mathbb{R}^{n}\right)$ of equation (1.1) such that

$$
x(t)=z_{-}\left(v_{-}, x_{-}, t\right)+y_{-}(t),
$$

where $\left|\dot{y}_{-}(t)\right|+\left|y_{-}(t)\right| \rightarrow 0$, as $t \rightarrow-\infty$. The deflection $y_{-}$from "free" motion satisfies the equation

$$
y_{-}(t)=\int_{-\infty}^{t} \int_{-\infty}^{\sigma}\left(F\left(z_{-}\left(v_{-}, x_{-}, .\right)+y_{-}\right)-F^{l}\left(z_{-}\left(v_{-}, x_{-}, .\right)\right)\right)(\tau) d \tau d \sigma .
$$

One can adapt the study of the operator $A$ given in the previous Section to study the integral equation (3.5) in the complete metric space

$$
M_{R(|x|)}^{*}:=\left\{f \in C^{1}\left(\mathbb{R}, \mathbb{R}^{n}\right) \mid\|f\|_{*} \leq R(|x|)\right\},
$$

where

$$
R(|x|):=\frac{2^{\frac{7}{2}} n^{\frac{3}{2}} \beta}{\alpha\left(\frac{1}{2}+\frac{|x|}{\sqrt{2}}\right)^{\alpha}}, R^{\prime}:=R_{0}(|x|)+R(|x|),
$$

and

$$
\begin{aligned}
\|f\|_{*}= & \max \left(\sup _{t \in(-\infty, 0)} \max \left(1,\left(\frac{1}{2}+\frac{\left|x_{-}\right|}{\sqrt{2}}+\left(\frac{\left|v_{-}\right|}{\sqrt{2}}-R^{\prime}\right)|t|\right)\right)|\dot{f}(t)| \sup _{(0,+\infty)}|\dot{f}|\right. \\
& \left.\left(\frac{\left|v_{-}\right|}{\sqrt{2}}-R^{\prime}\right) \sup _{(-\infty, 0)}|f|\right)
\end{aligned}
$$


Then one can prove that for $\left(v_{-}, x_{-}\right) \in \mathbb{R}^{n} \times \mathbb{R}^{n},<v_{-}, x_{-}>=0$ and

$$
\left|v_{-}\right| \geq \mu\left(\left|x_{-}\right|\right):=\mu_{l}\left(\left|x_{-}\right|\right)+10 \sqrt{2} R\left(\left|x_{-}\right|\right)+\frac{18 \sqrt{2} n \beta}{\alpha\left(\frac{1}{2}+\frac{\left|x_{-}\right|}{\sqrt{2}}\right)^{\alpha}},
$$

the unique solution $x$ of equation (1.1) that is characterized by (3.4) also satisfies the following asymptotics

$$
x(t)=z_{+}(\tilde{a}, \tilde{b}, t)+y_{+}(t),
$$

where $\left|\dot{y}_{+}(t)\right|+\left|y_{+}(t)\right| \rightarrow 0$, as $t \rightarrow+\infty$ for a unique $(\tilde{a}, \tilde{b}) \in \mathbb{R}^{n} \times \mathbb{R}^{n}$. The map $\tilde{S}$ defined by $\tilde{S}\left(v_{-}, x_{-}\right)=\left(v_{-}+\tilde{a}_{s c}, x_{-}+\tilde{b}_{s c}\right)$ on the set

$$
\mathcal{D}(\tilde{S}):=\left\{\left(v_{-}, x_{-}\right) \in \mathbb{R}^{n} \times \mathbb{R}^{n}\left|<v_{-}, x_{-}>=0,\right| v_{-} \mid \geq \mu\left(\left|x_{-}\right|\right)\right\}
$$

is our modified scattering map.

Then one can prove estimates for the modified scattering data $\left(\tilde{a}_{s c}, \tilde{b}_{s c}\right)$ that are similar to the estimates $(2.26)$ and (2.27) given in the previous Section. From these estimates on $\left(\tilde{a}_{s c}, \tilde{b}_{s c}\right)$ we derive the asymptotics of the scattering data as $|x| \rightarrow+\infty$ as well as their high energies asymptotics and their Born approximation at fixed energy. For their asymptotics as $|x| \rightarrow+\infty$ we have

$\tilde{a}_{s c}(\rho \theta, x)=\int_{\mathbb{R}} F^{l}\left(z_{-}(\rho \theta, x,).\right)(\tau) d \tau+\int_{\mathbb{R}} B^{s}(\tau \theta+x) \theta d \tau-\rho^{-1} P\left(\nabla V^{s}\right)(\theta, x)+O\left(|x|^{-2 \alpha-1}\right)$,

and

$$
\begin{aligned}
& \rho \tilde{b}_{s c}(\rho \theta, x)=\int_{-\infty}^{0} \int_{-\infty}^{\sigma} B^{s}(\tau \theta+x) \theta d \tau d \sigma-\int_{0}^{+\infty} \int_{\sigma}^{+\infty} B^{s}(\tau \theta+x) \theta d \tau d \sigma \\
& -\rho^{-1}\left(\int_{-\infty}^{0} \int_{-\infty}^{\sigma} \nabla V^{s}(\tau \theta+x) d \tau d \sigma-\int_{0}^{+\infty} \int_{\sigma}^{+\infty} \nabla V^{s}(\tau \theta+x) d \tau d \sigma\right)+O\left(|x|^{-2 \alpha}\right)
\end{aligned}
$$

for $\rho \in\left(\frac{1}{\sqrt{n}},+\infty\right)$ and for $\theta \in \mathbb{S}^{n-1},\langle\theta, x>=0$. In addition the modified scattering data have the following high energies asymptotics: for $(\theta, x) \in$ $T \mathbb{S}^{n-1}$

$\tilde{a}_{s c}(\rho \theta, x)=\int_{-\infty}^{+\infty} B(\tau \theta+x) \theta d \tau+\rho^{-1}\left(-P(\nabla V)(\theta, x)+\tilde{W}_{1}\left(B^{l}, B^{s}, \theta, x\right)\right)+o\left(\rho^{-1}\right)$,

as $\rho \rightarrow+\infty$, and 


$$
\begin{aligned}
\rho \tilde{b}_{s c}(\rho \theta, x)= & \int_{-\infty}^{0} \int_{-\infty}^{\sigma} B^{s}(\tau \theta+x) \theta d \tau d \sigma-\int_{0}^{+\infty} \int_{\sigma}^{+\infty} B^{s}(\tau \theta+x) \theta d \tau d \sigma \\
& +\rho^{-1}\left(-\int_{-\infty}^{0} \int_{-\infty}^{\sigma} \nabla V^{s}(\tau \theta+x) d \tau d \sigma+\int_{0}^{+\infty} \int_{\sigma}^{+\infty} \nabla V^{s}(\tau \theta+x) d \tau d \sigma\right. \\
& \left.+\tilde{W}_{2}\left(B^{l}, B^{s}, \theta, x\right)\right)+o\left(\rho^{-1}\right),
\end{aligned}
$$

as $\rho \rightarrow+\infty$, where

$$
\begin{aligned}
\tilde{W}_{1}\left(B^{l}, B^{s}, \theta, x\right)= & \int_{\mathbb{R}} B(\tau \theta+x)\left(\int_{-\infty}^{\tau} B(\sigma \theta+x) \theta d \sigma\right) d \tau \\
& +\sum_{k=1}^{n} \theta_{k} \int_{\mathbb{R}}\left(<\nabla B_{j, k}(\tau \theta+x), \int_{-\infty}^{\tau} \int_{-\infty}^{\sigma} B^{s}(\eta \theta+x) \theta d \eta d \sigma\right. \\
& \left.+\int_{0}^{\tau} \int_{-\infty}^{\sigma} B^{l}(\eta \theta+x) \theta d \eta d \sigma>\right)_{j=1 \ldots n} d \tau
\end{aligned}
$$

and

$$
\begin{aligned}
& \tilde{W}_{2}\left(B^{l}, B^{s}, \theta, x\right)=\int_{-\infty}^{0} \int_{-\infty}^{\sigma} B^{s}(\tau \theta+x)\left(\int_{-\infty}^{\tau} B(\eta \theta+x) \theta d \eta\right) d \tau d \sigma \\
& -\int_{0}^{+\infty} \int_{\sigma}^{+\infty} B^{s}(\tau \theta+x)\left(\int_{-\infty}^{\tau} B(\eta \theta+x) \theta d \eta\right) d \tau d \sigma \\
& +\sum_{k=1}^{n} \theta_{k}\left(\int_{-\infty}^{0} \int_{-\infty}^{\sigma}<\nabla B_{j, k}(\tau \theta+x), \int_{-\infty}^{\tau} \int_{-\infty}^{\eta_{1}} B^{s}\left(\eta_{2} \theta+x\right) \theta d \eta_{2} d \eta_{1}>d \tau d \sigma\right. \\
& +\int_{-\infty}^{0} \int_{-\infty}^{\sigma}<\nabla B_{j, k}^{s}(\tau \theta+x), \int_{0}^{\tau} \int_{-\infty}^{\eta_{1}} B^{l}\left(\eta_{2} \theta+x\right) \theta d \eta_{2} d \eta_{1}>d \tau d \sigma \\
& -\int_{0}^{+\infty} \int_{\sigma}^{+\infty}<\nabla B_{j, k}^{l}(\tau \theta+x), \int_{\tau}^{+\infty} \int_{\eta_{1}}^{+\infty} B^{s}\left(\eta_{2} \theta+x\right) \theta d \eta_{2} d \eta_{1}>d \tau d \sigma \\
& -\int_{0}^{+\infty} \int_{\sigma}^{+\infty}<\nabla B_{j, k}^{s}(\tau \theta+x), \int_{0}^{\tau} \int_{-\infty}^{\eta_{1}} B^{l}\left(\eta_{2} \theta+x\right) \theta d \eta_{2} d \eta_{1} \\
& \left.+\int_{-\infty}^{\tau} \int_{-\infty}^{\eta_{1}} B^{s}\left(\eta_{2} \theta+x\right) \theta d \eta_{2} d \eta_{1}>d \tau d \sigma\right)_{j=1 \ldots n} .
\end{aligned}
$$

For the Born approximation at fixed energy of the modified scattering data we have

$$
\tilde{a}_{s c}(\rho \theta, x)=\int_{-\infty}^{+\infty} B(\tau \theta+x) \theta d \tau-\rho^{-1} P(\nabla V)(\theta, x)+O\left(\beta^{2}\right),
$$


and

$$
\begin{aligned}
& \rho \tilde{b}_{s c}(\rho \theta, x)=\int_{-\infty}^{0} \int_{-\infty}^{\sigma} B^{s}(\tau \theta+x) \theta d \tau d \sigma-\int_{0}^{+\infty} \int_{\sigma}^{+\infty} B^{s}(\tau \theta+x) \theta d \tau d \sigma(3.17) \\
& -\rho^{-1}\left(\int_{-\infty}^{0} \int_{-\infty}^{\sigma} \nabla V^{s}(\tau \theta+x) d \tau d \sigma-\int_{0}^{+\infty} \int_{\sigma}^{+\infty} \nabla V^{s}(\tau \theta+x) d \tau d \sigma\right)+O\left(\beta^{2}\right),
\end{aligned}
$$
as $\beta \rightarrow 0^{+}$, and for $\rho \in\left(\frac{1}{\sqrt{n}},+\infty\right)$ and for $(\theta, x) \in T \mathbb{S}^{n-1}$.

Then results on the recovery of the short range part of the electromagnetic field from the high energies asymptotics or the Born approximation at fixed energy of the modified scattering data are similar to those given in Introduction (after Theorem 1.1) and at the end of Section 2.

\section{Preliminary estimates and proof of Lemma 2.2}

In the rest of the paper we use the following properties of the forces $\left(F^{l}, F^{s}\right)$ :

$$
\begin{gathered}
\left|F^{l}(x, v)\right| \leq \beta_{1}^{l} \sqrt{n}(1+|x|)^{-\alpha-1}(1+\sqrt{n}|v|), \\
\left|F^{s}(x, v)\right| \leq \beta_{2}^{s} \sqrt{n}(1+|x|)^{-\alpha-2}(1+\sqrt{n}|v|), \\
\left|F^{l}(x, v)-F^{l}\left(x^{\prime}, v^{\prime}\right)\right| \leq n \beta_{1}^{l}\left|v-v^{\prime}\right| \sup _{\varepsilon \in(0,1)}\left(1+\left|(1-\varepsilon) x+\varepsilon x^{\prime}\right|\right)^{-\alpha-1} \\
+n \beta_{2}^{l}\left|x-x^{\prime}\right| \sup _{\varepsilon \in(0,1)}\left(1+\left|(1-\varepsilon) x+\varepsilon x^{\prime}\right|\right)^{-\alpha-2}\left(1+\sqrt{n}\left|(1-\varepsilon) v+\varepsilon v^{\prime}\right|\right), \\
\left|F^{s}(x, v)-F^{s}\left(x^{\prime}, v^{\prime}\right)\right| \leq n \beta_{2}^{s}\left|v-v^{\prime}\right| \sup _{\varepsilon \in(0,1)}\left(1+\left|(1-\varepsilon) x+\varepsilon x^{\prime}\right|\right)^{-\alpha-2} \\
+n \beta_{3}^{s}\left|x-x^{\prime}\right| \sup _{\varepsilon \in(0,1)}\left(1+\left|(1-\varepsilon) x+\varepsilon x^{\prime}\right|\right)^{-\alpha-3}\left(1+\sqrt{n}\left|(1-\varepsilon) v+\varepsilon v^{\prime}\right|\right),
\end{gathered}
$$

for $\left(x, x^{\prime}, v, v^{\prime}\right) \in\left(\mathbb{R}^{n}\right)^{4}$.

In the rest of this section we first prove the existence and uniqueness of the solutions $z_{+}$(similarly one can prove the existence and uniqueness of $z_{-}$). Then we give some properties of the solutions $z_{-}$. Finally we prove Lemma 2.2 .

Let us prove the existence and uniqueness of the solutions $z_{+}$. Let $v \in \mathbb{R}^{n}$, $|v| \geq \mu^{l}$. Let $\mathcal{V}$ be the complete metric space defined by

$$
\mathcal{V}:=\left\{f \in C^{1}\left(\mathbb{R}, \mathbb{R}^{n}\right) \mid f(0)=0 \text { and } \sup _{\mathbb{R}}|\dot{f}| \leq R_{0},\right\}
$$


endowed with the following norm $\|f\|_{\mathcal{V}}:=\sup _{\mathbb{R}}|\dot{f}|$, where $R_{0}$ is defined in (1.7). Note that $\mathcal{V}$ is a convex subset of $C^{1}\left(\mathbb{R}, \mathbb{R}^{n}\right)$. For $f \in \mathcal{V}$ we define $G(f) \in C^{2}\left(\mathbb{R}, \mathbb{R}^{n}\right)$ by

$$
G(f)(0)=0, \dot{G}(f)(t):=-\int_{t}^{+\infty} F^{l}(. v+f)(\tau) d \tau \text { for } t \in \mathbb{R} .
$$

We use the following estimate (4.6)

$$
|\tau v+f(\tau)| \geq|\tau v|-|f(\tau)| \geq\left(|v|-R_{0}\right)|\tau|,
$$

for $\tau \in \mathbb{R}$ and $f \in \mathcal{V}$. Using (4.1) and (4.6) we obtain that

$$
|\dot{G}(f)(t)| \leq \beta_{1}^{l} \sqrt{n} \int_{t}^{+\infty} \frac{(1+\sqrt{n}|v+\dot{f}(\tau)|) d \tau}{\left(1+\left(|v|-R_{0}\right)|\tau|\right)^{\alpha+1}} \leq \frac{2 \beta_{1}^{l} \sqrt{n}\left(1+\sqrt{n}\left(|v|+R_{0}\right)\right)}{\alpha\left(|v|-R_{0}\right)} \leq R_{0}
$$

for $t \in \mathbb{R}$ (we used the definition of $R_{0}$ and the inequality $|v| \geq \mu^{l}$ ).

Now let $\left(f_{1}, f_{2}\right) \in \mathcal{V}^{2}$. Using (4.3) and (4.6) we have

$$
\begin{gathered}
\left|F^{l}\left(. v+f_{1}\right)-F^{l}\left(. v+f_{2}\right)\right|(\tau) \leq \frac{n \beta_{1}^{l}}{\left(1+\left(|v|-R_{0}\right)|\tau|\right)^{\alpha+1}} \sup _{\mathbb{R}}\left|\dot{f}_{1}-\dot{f}_{2}\right| \\
+\frac{n \beta_{2}^{l}\left(1+\sqrt{n}\left(|v|+R_{0}\right)\right)|\tau|}{\left(1+\left(|v|-R_{0}\right)|\tau|\right)^{\alpha+2}} \sup _{s \in \mathbb{R} \backslash\{0\}} \frac{\left|\left(f_{1}-f_{2}\right)(s)\right|}{|s|} \\
\leq \frac{n \beta_{1}^{l}+\frac{n \beta_{2}^{l}}{\left(|v|-R_{0}\right)}\left(1+\sqrt{n}\left(|v|+R_{0}\right)\right)}{\left(1+\left(|v|-R_{0}\right)|\tau|\right)^{\alpha+1}}\left\|f_{1}-f_{2}\right\|_{\mathcal{V}}
\end{gathered}
$$

for $\tau \in \mathbb{R}$. Therefore we obtain

$$
\left|\dot{G}\left(f_{1}\right)(t)-\dot{G}\left(f_{2}\right)(t)\right| \leq 2 n \frac{\beta_{1}^{l}+\frac{\beta_{2}^{l}}{\left(|v|-R_{0}\right)}\left(1+\sqrt{n}\left(|v|+R_{0}\right)\right)}{\alpha\left(|v|-R_{0}\right)}\left\|f_{1}-f_{2}\right\|_{\mathcal{V}}
$$

for $t \in \mathbb{R}$.

From (4.7), (4.9) and condition $|v| \geq \mu^{l}$ it follows that the operator $G$ is a $\frac{1}{2}$-contraction map from $\mathcal{V}$ to $\mathcal{V}$. Set $z_{+}(v, t)=t v+f(t)$ for $t \in \mathbb{R}$, where $f$ denotes the unique fixed point of $G$ in $\mathcal{V}$. Then $z_{+}(v,$.$) satisfies (1.8),(1.9)$, (1.10).

We will use the following properties of the solutions $z_{ \pm}$.

Lemma 4.1. Let $(\rho, \theta) \in\left[\mu^{l},+\infty\right) \times \mathbb{S}^{n-1}$. Then we have

$$
\left|z_{ \pm}\left(\rho \theta, \frac{s}{\rho}\right)-s \theta\right| \leq \frac{R_{0}|s|}{\rho}, \text { for } s \in \mathbb{R} .
$$


In addition for $(s, \theta) \in \mathbb{R} \times \mathbb{S}^{n-1}$ we have

$$
\lim _{\rho \rightarrow+\infty} \rho\left(z_{-}\left(\rho \theta, \frac{s}{\rho}\right)-s \theta\right)=\int_{0}^{s} \int_{-\infty}^{\sigma} B^{l}(u \theta) \theta d u d \sigma .
$$

Proof of Lemma 4.1. The estimate (4.10) follows from (1.9) and (1.10). The limit (4.11) follows from (1.10), (4.10) and the identity

$\rho\left(z_{-}\left(\rho \theta, \frac{s}{\rho}\right)-s \theta\right)=\int_{0}^{s} \int_{-\infty}^{\sigma} \rho^{-1}\left(-\nabla V^{l}\left(z_{-}\left(\rho \theta, \frac{u}{\rho}\right)\right)+B^{l}\left(z_{-}\left(\rho \theta, \frac{u}{\rho}\right)\right) \dot{z}_{-}\left(\rho \theta, \frac{u}{\rho}\right)\right) d u d \sigma$,

that holds for $(\rho, \theta) \in\left[\mu^{l},+\infty\right) \times \mathbb{S}^{n-1}$.

To prove Lemma 2.2 we need the following standard lemma.

Lemma 4.2. Let $x \in C^{2}\left(\mathbb{R}, \mathbb{R}^{n}\right)$ satisfy equation (1.1) and let $z \in C^{2}\left(\mathbb{R}, \mathbb{R}^{n}\right)$ satisfy equation (1.8). Assume that there exists $v \in \mathbb{R}^{n}, v \neq 0$, so that $\dot{x}(t) \rightarrow v$ and $\dot{z}(t) \rightarrow v$ as $t \rightarrow+\infty$. Then

$$
\sup _{(0,+\infty)}|x-z|<\infty \text { and } \sup _{t \in(0,+\infty)}(1+t)|\dot{x}-\dot{z}|(t)<\infty .
$$

Proof of Lemma 2.2. We need the following preliminary estimate (4.16). Observe that for $f \in M_{R, r}$

$$
\sup _{(-\infty, 0)}|f| \leq \frac{R}{\frac{\left|v_{-}\right|}{\sqrt{2}}-R^{\prime}} \leq r,
$$
that

From the formula $g(\tau)=g(0)+\int_{0}^{\tau} \dot{g}(s) d s$ for $g \in C^{1}\left(\mathbb{R}, \mathbb{R}^{n}\right)$ it follows

$$
\left|\left(f_{1}-f_{2}\right)(\tau)\right| \leq \sup _{(-\infty, 0)}\left|f_{1}-f_{2}\right|+|\tau| \sup _{(0,+\infty)}\left|\dot{f}_{1}-\dot{f}_{2}\right| \text { for } \tau \in \mathbb{R},
$$

and for $\left(f_{1}, f_{2}\right) \in M_{R, r}^{2}$. Hence

$\left|z_{-}\left(v_{-}, \tau\right)+x+f(\tau)\right| \geq\left|x+\tau v_{-}\right|-\left|z_{-}\left(v_{-}, \tau\right)-\tau v_{-}\right|-|f(\tau)| \geq \frac{|x|}{\sqrt{2}}-r+|\tau|\left(\frac{\left|v_{-}\right|}{\sqrt{2}}-R^{\prime}\right)$

for $(f, \tau) \in M_{R, r} \times \mathbb{R}$ and for any $x \in \mathbb{R}^{n}$ so that $\left\langle x, v_{-}>=0\right.$. We used the inequality $\left|x+\tau v_{-}\right| \geq \frac{|x|}{\sqrt{2}}+|\tau| \frac{\left|v_{-}\right|}{\sqrt{2}}\left(<x, v_{-}>=0\right.$ ) and (4.15) (for $\left(f_{1}, f_{2}\right)=(f, 0)$ and $\left.f \in M_{R, r}\right),(4.14)$ and $(1.10)$.

Hence the integral $\int_{-\infty}^{+\infty} F\left(z_{-}\left(v_{-},.\right)+x_{-}+f\right)(\tau) d \tau$ is absolutely convergent for any $f \in M_{R, r}$. And when $y_{-} \in M_{R, r}$ is a fixed point for $A$ then $z_{-}\left(v_{-},.\right)+$ 
$x_{-}+y_{-}$satisfies equation (1.1) (see (2.2) and (2.3)) and $\dot{z}_{-}\left(v_{-}, t\right)+\dot{y}_{-}(t)=$ $\int_{-\infty}^{t} F\left(z_{-}\left(v_{-},.\right)+x_{-}+y_{-}\right)(\tau) d \tau \rightarrow a\left(v_{-}, x_{-}\right)$as $t \rightarrow+\infty$, where $a\left(v_{-}, x_{-}\right)$is defined in (2.10). Then from Lemma 4.2 it follows that $\sup _{(0,+\infty)} \mid z_{-}\left(v_{-},.\right)+$ $x_{-}+y_{-}-z_{+}\left(a\left(v_{-}, x_{-}\right),.\right) \mid<+\infty$ and $\sup _{t \in(0,+\infty)}(1+t) \mid \dot{z}_{-}\left(v_{-}, t\right)+\dot{y}_{-}(t)-$ $\dot{z}_{+}\left(a\left(v_{-}, x_{-}\right), t\right) \mid<\infty$, and the integral on the right hand side of (2.12) is absolutely convergent. Then the decomposition (2.9) follows from the equality $A\left(y_{-}\right)=y_{-}$and straightforward computations.

\section{$5 \quad$ Proof of Lemma 2.1}

We shorten $z_{-}\left(v_{-},.\right)$to $z_{-}$in this paragraph.

We first prove the estimates (5.4), (5.5) and (5.7) given below. Let $f \in$ $M_{R, r}$. Using (4.2) and (4.16) we have

$$
\left|F^{s}\left(z_{-}+\varepsilon x_{-}+f\right)(\tau)\right| \leq \frac{\sqrt{n} \beta_{2}\left(1+\sqrt{n}\left(\left|v_{-}\right|+R^{\prime}\right)\right)}{\left(1+\frac{\varepsilon\left|x_{-}\right|}{\sqrt{2}}-r+|\tau|\left(\frac{v_{-} \mid}{\sqrt{2}}-R^{\prime}\right)\right)^{\alpha+2}},
$$

for $\tau \in \mathbb{R}$ and for $\varepsilon \in[0,1]$. Then from (2.3) it follows that

$$
|\dot{A}(f)(t)| \leq\left|\int_{-\infty}^{t}\left(F\left(z_{-}+x_{-}+f\right)(\tau)-F^{l}\left(z_{-}\right)(\tau)\right) d \tau\right|
$$

for $t \in \mathbb{R}$. Using (4.3) and (4.16) we obtain

$$
\begin{aligned}
& \left|F^{l}\left(z_{-}+x_{-}+f_{1}\right)(\tau)-F^{l}\left(z_{-}+\mu x_{-}+f_{2}\right)(\tau)\right| \leq \frac{n \beta_{1}^{l}\left|\dot{f}_{1}-\dot{f}_{2}\right|(\tau)}{\left(1-r+\mu \frac{\left|x_{-}\right|}{\sqrt{2}}+\left(\frac{\left|v_{-}\right|}{\sqrt{2}}-R^{\prime}\right)|\tau|\right)^{\alpha+1}} \\
& +\frac{n \beta_{2}\left((1-\mu)\left|x_{-}\right|+\left|f_{1}-f_{2}\right|(\tau)\right)\left(1+\sqrt{n} \sup _{\varepsilon \in(0,1)}\left|\dot{z}_{-}+\varepsilon \dot{f}_{1}+(1-\varepsilon) \dot{f}_{2}\right|\right)}{\left(1-r+\mu \frac{|x-|}{\sqrt{2}}+\left(\frac{\left|v_{-}\right|}{\sqrt{2}}-R^{\prime}\right)|\tau|\right)^{\alpha+2}}
\end{aligned}
$$

for $\left(f_{1}, f_{2}\right) \in M_{R, r}^{2}$ and for $(\tau, \mu) \in \mathbb{R} \times[0,1]$. We integrate (5.1) and (5.3) over $(-\infty, t)$, and we use the estimates $|f(\tau)| \leq r$ and $|\dot{f}(\tau)| \leq R(1-r+$ $\left.\left(\frac{\left|v_{-}\right|}{\sqrt{2}}-R^{\prime}\right)|\tau|\right)^{-1}$ for $\tau \leq 0, \sup _{(-\infty, 0)}|\dot{f}| \leq R$, and we obtain

$$
\begin{aligned}
& |\dot{A}(f)(t)| \leq \frac{n \beta_{1}^{l} R+\sqrt{n} \beta_{2}\left(\sqrt{n}\left(\left|x_{-}\right|+r\right)+1\right)\left(1+\sqrt{n}\left(\left|v_{-}\right|+R^{\prime}\right)\right)}{(\alpha+1)\left(\frac{\left|v_{-}\right|}{\sqrt{2}}-R^{\prime}\right)\left(1-r-\left(\frac{\left|v_{-}\right|}{\sqrt{2}}-R^{\prime}\right) t\right)^{\alpha+1}}, \\
& |A(f)(t)| \leq \frac{n \beta_{1}^{l} R+\sqrt{n} \beta_{2}\left(\sqrt{n}\left(\left|x_{-}\right|+r\right)+1\right)\left(1+\sqrt{n}\left(\left|v_{-}\right|+R^{\prime}\right)\right)}{\alpha(\alpha+1)\left(\frac{\left|v_{-}\right|}{\sqrt{2}}-R^{\prime}\right)^{2}\left(1-r-\left(\frac{\left|v_{-}\right|}{\sqrt{2}}-R^{\prime}\right) t\right)^{\alpha}},
\end{aligned}
$$

for $t \leq 0$. 
Let $t \geq 0$ and $\left|v_{-}\right|>\sqrt{2} R^{\prime}$. Integrating (5.3) over $(0, t)$ and using the estimate $\sup _{(0,+\infty)}|\dot{f}| \leq R$, and (4.15) (for $\left(f_{1}, f_{2}\right)=(f, 0)$ ), we obtain

$$
\begin{aligned}
& \int_{0}^{t}\left|F^{l}\left(z_{-}+x_{-}+f\right)(\tau)-F^{l}\left(z_{-}\right)(\tau)\right| d \tau \\
\leq & \frac{n \beta_{1}^{l} R+n \beta_{2} \frac{R}{\frac{v_{-} \mid}{\sqrt{2}}-R^{\prime}}\left(1+\sqrt{n}\left(\left|v_{-}\right|+R^{\prime}\right)\right)}{\alpha\left(\frac{\left|v_{-}\right|}{\sqrt{2}}-R^{\prime}\right)(1-r)^{\alpha}}+\frac{n \beta_{2}\left(\left|x_{-}\right|+r\right)\left(1+\sqrt{n}\left(\left|v_{-}\right|+R^{\prime}\right)\right)}{(\alpha+1)\left(\frac{\left|v_{-}\right|}{\sqrt{2}}-R^{\prime}\right)(1-r)^{\alpha+1}} .
\end{aligned}
$$

Hence combining (5.2), (5.1) integrated over $(0, t),(5.6)$ and $(5.4)$ (at " $t=0 ")$ we obtain

$$
\begin{aligned}
|\dot{A}(f)(t)| & \leq \frac{n \beta_{1}^{l} R+n \beta_{2} \frac{R}{\frac{v_{-} \mid}{\sqrt{2}}-R^{\prime}}\left(1+\sqrt{n}\left(\left|v_{-}\right|+R^{\prime}\right)\right)}{\alpha\left(\frac{\left|v_{-}\right|}{\sqrt{2}}-R^{\prime}\right)(1-r)^{\alpha}} \\
+ & \frac{n \beta_{1}^{l} R+2 \sqrt{n} \beta_{2}\left(\sqrt{n}\left(\left|x_{-}\right|+r\right)+1\right)\left(1+\sqrt{n}\left(\left|v_{-}\right|+R^{\prime}\right)\right)}{(\alpha+1)\left(\frac{\left|v_{-}\right|}{\sqrt{2}}-R^{\prime}\right)(1-r)^{\alpha+1}}
\end{aligned}
$$

Then estimate (2.7) follows from (5.4), (5.5) and (5.7).

Now we prove the estimates $(5.10),(5.11)$ and (5.13) given below. Estimate (2.8) follows from those latter estimates. Let $\left|v_{-}\right|>\sqrt{2} R^{\prime}$, and let $\left(f_{1}, f_{2}\right) \in M_{R, r}^{2}$. From (4.4) and (4.16) it follows that

$$
\begin{aligned}
& \left|F^{s}\left(z_{-}+x_{-}+f_{1}\right)(\tau)-F^{s}\left(z_{-}+x_{-}+f_{2}\right)(\tau)\right| \leq \frac{n \beta_{2}\left|\dot{f}_{1}-\dot{f}_{2}\right|(\tau)}{\left(1-r+\frac{\left|x_{-}\right|}{\sqrt{2}}+\left(\frac{\left|v_{-}\right|}{\sqrt{2}}-R^{\prime}\right)|\tau|\right)^{\alpha+2}} \\
& +\frac{n \beta_{3}^{s}\left|f_{1}-f_{2}\right|(\tau)\left(1+\sqrt{n} \sup _{\varepsilon \in(0,1)}\left|\dot{z}_{-}+\varepsilon \dot{f}_{1}(\tau)+(1-\varepsilon) \dot{f}_{2}(\tau)\right|\right)}{\left(1-r+\frac{\left|x_{-}\right|}{\sqrt{2}}+\left(\frac{\left|v_{-}\right|}{\sqrt{2}}-R^{\prime}\right)|\tau|\right)^{\alpha+3}}
\end{aligned}
$$

for $\tau \in \mathbb{R}$. We integrate (5.8) over $(-\infty, t)$, and we have

$$
\begin{aligned}
& \int_{-\infty}^{t}\left|F^{s}\left(z_{-}+x_{-}+f_{1}\right)(\tau)-F^{s}\left(z_{-}+x_{-}+f_{2}\right)(\tau)\right| d \tau \\
& \leq \int_{-\infty}^{t} \frac{n \beta_{2} \sup _{s \in(-\infty, 0)}\left(1-r+\left(\frac{\left|v_{-}\right|}{\sqrt{2}}-R^{\prime}\right)|s|\right)\left|\dot{f}_{1}-\dot{f}_{2}\right|(s) d \tau}{\left(1-r+\left(\frac{\left|v_{-}\right|}{\sqrt{2}}-R^{\prime}\right)|\tau|\right)^{\alpha+3}} \\
& \quad+\int_{-\infty}^{t} \frac{n \beta_{3}^{s}\left(1+\sqrt{n}\left(\left|v_{-}\right|+R^{\prime}\right)\right) \sup _{(-\infty, 0)}\left|f_{1}-f_{2}\right| d \tau}{\left(1-r+\left(\frac{\left|v_{-}\right|}{\sqrt{2}}-R^{\prime}\right)|\tau|\right)^{\alpha+3}} \\
& \quad \leq \frac{n\left(\beta_{2}+\beta_{3}^{s} \frac{1+\sqrt{n}\left(\left|v_{-}\right|+R^{\prime}\right)}{\frac{\mid v_{-}}{\sqrt{2}}-R^{\prime}}\right) \| f_{1}-f_{2}||}{(\alpha+2)\left(\frac{\left|v_{-}\right|}{\sqrt{2}}-R^{\prime}\right)\left(1-r+\left(\frac{\mid v_{-}-}{\sqrt{2}}-R^{\prime}\right)|t|\right)^{\alpha+2}}
\end{aligned}
$$


An estimate similar to (5.9) holds for $F^{l}$ in place of $F^{s}$, and combining these two latter estimates we obtain

$$
\begin{aligned}
& \left|\dot{A}\left(f_{1}\right)(t)-\dot{A}\left(f_{2}\right)(t)\right| \leq \frac{n\left\|f_{1}-f_{2}\right\|}{\left(\frac{\left|v_{-}\right|}{\sqrt{2}}-R^{\prime}\right)\left(1-r+\left(\frac{\left|v_{-}\right|}{\sqrt{2}}-R^{\prime}\right)|t|\right)^{\alpha+1}} \\
& \times\left(\frac{\beta_{1}^{l}+\beta_{2} \frac{1+\sqrt{n}\left(\left|v_{-}\right|+R^{\prime}\right)}{\frac{\left|v_{-}\right|}{\sqrt{2}}-R^{\prime}}}{(\alpha+1)}+\frac{\beta_{2}+\beta_{3}^{s \frac{1+\sqrt{n}\left(\left|v_{-}\right|+R^{\prime}\right)}{\frac{\mid v_{-}}{\sqrt{2}}-R^{\prime}}}}{(\alpha+2)\left(1-r+\left(\frac{\left|v_{-}\right|}{\sqrt{2}}-R^{\prime}\right)|t|\right)}\right), \\
& \left|A\left(f_{1}\right)(t)-A\left(f_{2}\right)(t)\right| \leq \frac{n\left\|f_{1}-f_{2}\right\|}{(\alpha+1)\left(\frac{\left|v_{-}\right|}{\sqrt{2}}-R^{\prime}\right)^{2}\left(1-r+\left(\frac{\left|v_{-}\right|}{\sqrt{2}}-R^{\prime}\right)|t|\right)^{\alpha}} \\
& \times\left(\frac{\beta_{1}^{l}+\beta_{2} \frac{1+\sqrt{n}\left(\left|v_{-}\right|+R^{\prime}\right)}{\frac{|v-|}{\sqrt{2}}-R^{\prime}}}{\alpha}+\frac{\beta_{2}+\beta_{3}^{s \frac{1+\sqrt{n}\left(\left|v_{-}\right|+R^{\prime}\right)}{\frac{\left|v_{-}\right|}{\sqrt{2}}-R^{\prime}}}}{(\alpha+2)\left(1-r+\left(\frac{\left|v_{-}\right|}{\sqrt{2}}-R^{\prime}\right)|t|\right)}\right) .
\end{aligned}
$$

Let $t \geq 0$ and $\left|v_{-}\right|>\sqrt{2} R^{\prime}, r<1$, and let $\left(f_{1}, f_{2}\right) \in M_{R, r}^{2}$. We integrate (5.8) over $(0, t)$, and we use the estimate (4.15), and we have

$$
\begin{aligned}
& \int_{0}^{t}\left|F^{s}\left(z_{-}+x_{-}+f_{1}\right)(\tau)-F^{s}\left(z_{-}+x_{-}+f_{2}\right)(\tau)\right| d \tau \leq \frac{n \beta_{2} \sup _{(0,+\infty)}\left|\dot{f}_{1}-\dot{f}_{2}\right|}{(\alpha+1)\left(\frac{\left|v_{-}\right|}{\sqrt{2}}-R^{\prime}\right)\left(1-r+\frac{\left|x_{-}\right|}{\sqrt{2}}\right)^{\alpha+1}} \\
& +\frac{\left.n \beta_{3}^{s} \sup _{(-\infty, 0)}\left|f_{1}-f_{2}\right|\left(1+\sqrt{n}\left(\left|v_{-}\right|+R^{\prime}\right)\right)\right)}{(\alpha+2)\left(\frac{\left|v_{-}\right|}{\sqrt{2}}-R^{\prime}\right)\left(1-r+\frac{\left|x_{-}\right|}{\sqrt{2}}\right)^{\alpha+2}} \\
& +\frac{\left.n \beta_{3}^{s} \sup _{(0,+\infty)}\left|\dot{f}_{1}-\dot{f}_{2}\right|\left(1+\sqrt{n}\left(\left|v_{-}\right|+R^{\prime}\right)\right)\right)}{(\alpha+1)\left(\frac{\left|v_{-}\right|}{\sqrt{2}}-R^{\prime}\right)^{2}\left(1-r+\frac{|x-|}{\sqrt{2}}\right)^{\alpha+1}} .
\end{aligned}
$$

A similar estimate holds for $F^{l}$ in place of $F^{s}$. Then combining these two latter estimates and (5.10) (at " $t=0 ")$, we obtain

$$
\begin{aligned}
& \left|\dot{A}\left(f_{1}\right)(t)-\dot{A}\left(f_{2}\right)(t)\right| \leq \frac{n\left\|f_{1}-f_{2}\right\|}{\left(\frac{\left|v_{-}\right|}{\sqrt{2}}-R^{\prime}\right)(1-r)^{\alpha}}\left(\frac{\beta_{2}}{(\alpha+2)(1-r)^{2}}\right. \\
& +\frac{\beta_{2}+\beta_{1}^{l}}{(\alpha+1)(1-r)}+\frac{\beta_{1}^{l}}{\alpha}+\frac{1+\sqrt{n}\left(\left|v_{-}\right|+R^{\prime}\right)}{\left(\frac{\left|v_{-}\right|}{\sqrt{2}}-R^{\prime}\right)} \\
& \left.\times\left(\frac{2 \beta_{3}^{s}}{(\alpha+2)(1-r)^{2}}+\frac{\beta_{3}^{s}+2 \beta_{2}}{(\alpha+1)(1-r)}+\frac{\beta_{2}}{\alpha}\right)\right) .
\end{aligned}
$$




\section{Proof of Theorems 1.1 and 2.4}

Proof of Theorem 2.4. We shorten $z_{-}\left(v_{-},.\right), a_{s c}\left(v_{-}, x_{-}\right), a\left(v_{-}, x_{-}\right)$and $b_{s c}\left(v_{-}, x_{-}\right)$ to $z_{-}, a_{s c}, a$ and $b_{s c}$ in this proof. Computations similar to (5.4) show that

$$
\begin{aligned}
\sup _{(-\infty, t)}\left|\dot{y}_{-}\right|= & \sup _{(-\infty, t)}\left|\dot{A}\left(y_{-}\right)\right| \leq \frac{n \beta_{1}^{l} \sup _{(-\infty, t)}\left|\dot{y}_{-}\right|}{\alpha\left(\frac{\left|v_{-}\right|}{\sqrt{2}}-R^{\prime}\right)(1-r)^{\alpha}} \\
& +\frac{\sqrt{n} \beta_{2}\left(\sqrt{n}\left(\left|x_{-}\right|+r\right)+1\right)\left(1+\sqrt{n}\left(\left|v_{-}\right|+R^{\prime}\right)\right)}{(\alpha+1)\left(\frac{\left|v_{-}\right|}{\sqrt{2}}-R^{\prime}\right)\left(1-r-\left(\frac{\left|v_{-}\right|}{\sqrt{2}}-R^{\prime}\right) t\right)^{\alpha}} .
\end{aligned}
$$

Hence using condition (2.16) we obtain

$$
\frac{1}{2} \sup _{(-\infty, t)}\left|\dot{y}_{-}\right| \leq \frac{n \beta_{2}\left(\left|x_{-}\right|+2\right)\left(1+\sqrt{n}\left(\left|v_{-}\right|+R^{\prime}\right)\right)}{(\alpha+1)\left(\frac{\left|v_{-}\right|}{\sqrt{2}}-R^{\prime}\right)\left(1-r-\left(\frac{v_{-} \mid}{\sqrt{2}}-R^{\prime}\right) t\right)^{\alpha+1}},
$$

which gives (2.21) (we also use (2.13)). Computations similar to (5.7) show that

$$
\begin{aligned}
\sup _{\mathbb{R}}\left|\dot{y}_{-}\right| \leq & \sup _{\mathbb{R}}\left|\dot{y}_{-}\right| \frac{\left(2 n \beta_{1}^{l}+\frac{n \beta_{2}}{\frac{1 v_{-} \mid}{\sqrt{2}}-R^{\prime}}\left(1+\sqrt{n}\left(\left|v_{-}\right|+R^{\prime}\right)\right)\right)}{\alpha\left(\frac{\left|v_{-}\right|}{\sqrt{2}}-R^{\prime}\right)(1-r)^{\alpha}} \\
& +\frac{2 \sqrt{n} \beta_{2}\left(\sqrt{n}\left(\left|x_{-}\right|+r\right)+1\right)\left(1+\sqrt{n}\left(\left|v_{-}\right|+R^{\prime}\right)\right)}{(\alpha+1)\left(\frac{\left|v_{-}\right|}{\sqrt{2}}-R^{\prime}\right)(1-r)^{\alpha+1}} .
\end{aligned}
$$

Then we use condition (2.16) and (2.13), and we obtain (2.22). The estimate (2.23) follows by integrating over $\mathbb{R}$ both sides of the estimate $(5.1)("(\varepsilon, f)=$ $\left.\left(1, y_{-}\right) "\right)$ and both sides of an estimate similar to (5.1) with $F^{l}$ in place of $F^{s}$.

Now we prove (2.24) and (2.25). We rewrite $y_{+}$as follows

$$
\dot{y}_{+}=h_{0}+h_{1},
$$

where

$h_{0}(t):=-\int_{t}^{+\infty} F^{s}\left(z_{-}+x_{-}+y_{-}\right)(\tau) d \tau, h_{1}(t):=-\int_{t}^{+\infty}\left(F^{l}\left(z_{-}+x_{-}+y_{-}\right)-F^{l}\left(z_{+}(a,).\right)\right)(\tau) d \tau$

for $t \geq 0$. We estimate $\dot{h}_{0}$. We also need the following estimate (6.7). For $\varepsilon, \varepsilon^{\prime} \in(0,1)$ and $\tau \geq 0$ we have

$$
\begin{aligned}
& \left|(1-\varepsilon)\left(z_{-}(\tau)+y_{-}(\tau)\right)+\varepsilon z_{+}(a, \tau)+\varepsilon^{\prime} x_{-}\right| \\
\geq & \left|\varepsilon^{\prime} x_{-}+\tau v_{-}\right|-(1-\varepsilon)\left|z_{-}(\tau)-\tau v_{-}\right|-(1-\varepsilon) R \tau-r \\
& -\varepsilon\left|z_{+}(\tau)-\tau a\right|-\varepsilon\left|a_{s c}\right| \tau \\
\geq & \varepsilon^{\prime} \frac{\left|x_{-}\right|}{\sqrt{2}}-r+\tau\left(\frac{\left|v_{-}\right|}{\sqrt{2}}-R_{0}+(1-\varepsilon) R\right)-\varepsilon\left|a_{s c}\right| \tau .
\end{aligned}
$$


We used (1.10) for " $v "=v_{-}$and for " $v "=a$ (by conservation of the energy $\left.\left|v_{-}\right|=a\right)$. Then we use (2.16), (6.6) and (2.23), and we have for $\left(\varepsilon, \varepsilon^{\prime}\right) \in$ $(0,1)^{2}$ and $\tau \geq 0$

$$
\left|(1-\varepsilon)\left(z_{-}(\tau)+y_{-}(\tau)\right)+\varepsilon z_{+}(a, \tau)+\varepsilon^{\prime} x_{-}\right| \geq \varepsilon^{\prime} \frac{\left|x_{-}\right|}{\sqrt{2}}-r+\tau\left(\frac{\left|v_{-}\right|}{\sqrt{2}}-R^{\prime}\right) .
$$

From (5.1) it follows that

$$
\begin{aligned}
\left|h_{0}(t)\right| & \leq \int_{t}^{+\infty}\left|F^{s}\left(z_{-}+x_{-}+y_{-}\right)\right|(\tau) d \tau \\
& \leq \frac{\beta_{2}^{s} \sqrt{n}\left(1+\sqrt{n}\left(\left|v_{-}\right|+R^{\prime}\right)\right)}{(\alpha+1)\left(\frac{\left|v_{-}\right|}{\sqrt{2}}-R^{\prime}\right)\left(1+\frac{\left|x_{-}\right|}{\sqrt{2}}-r+\left(\frac{\left|v_{-}\right|}{\sqrt{2}}-R^{\prime}\right) t\right)^{\alpha+1}}
\end{aligned}
$$

for $t \geq 0$. Now set

$$
\delta_{1}(t):=\sup _{s \in(t,+\infty)}\left(1-r+\left(\frac{\left|v_{-}\right|}{\sqrt{2}}-R^{\prime}\right) s\right)\left|\dot{y}_{+}(s)\right|, \delta_{2}:=\sup _{(0,+\infty)}\left|b_{s c}+y_{+}\right|,
$$

for $t \geq 0$. We remind that $\delta_{1}(t)$ and $\delta_{2}$ are finite by Lemma 4.2 (for " $(x, z) "=$ $\left.\left(z_{-}+x_{-}+y_{-}, z_{+}(a,).\right)\right)$. Then we use (4.3) and (6.7), and we obtain

$$
\begin{aligned}
\left|h_{1}(t)\right| & \leq \int_{t}^{+\infty} \frac{n \beta_{1}^{l} \delta_{1}(t)+n \beta_{2}^{l}\left(\delta_{2}+\left|x_{-}\right|\right)\left(1+\sqrt{n}\left(\left|v_{-}\right|+R^{\prime}\right)\right)}{\left(1-r+\tau\left(\frac{\mid v_{-}}{\sqrt{2}}-R^{\prime}\right)\right)^{\alpha+2}} d \tau d \sigma \\
& \leq \frac{n \beta_{1}^{l} \delta_{1}(t)+n \beta_{2}^{l}\left(\delta_{2}+\left|x_{-}\right|\right)\left(1+\sqrt{n}\left(\left|v_{-}\right|+R^{\prime}\right)\right)}{(\alpha+1)\left(\frac{\left|v_{-}\right|}{\sqrt{2}}-R^{\prime}\right)\left(1-r+t\left(\frac{\left|v_{-}\right|}{\sqrt{2}}-R^{\prime}\right)\right)^{\alpha+1}}
\end{aligned}
$$

for $t \geq 0$. Hence combining (6.4), (6.8) and (6.10) and condition (2.16) we have

$$
\frac{\delta_{1}(t)}{2} \leq \frac{\left(n \beta_{2}^{l}\left(\delta_{2}+\left|x_{-}\right|\right)+\sqrt{n} \beta_{2}^{s}\right)\left(1+\sqrt{n}\left(\left|v_{-}\right|+R^{\prime}\right)\right)}{(\alpha+1)\left(\frac{\left|v_{-}\right|}{\sqrt{2}}-R^{\prime}\right)\left(1-r+t\left(\frac{\left|v_{-}\right|}{\sqrt{2}}-R^{\prime}\right)\right)^{\alpha}}
$$

for $t \geq 0$. In addition from (2.11) and (6.2) it follows that

$$
\left|b_{s c}+y_{+}(0)\right|=\left|y_{-}(0)\right| \leq \frac{2 n \beta_{2}\left(\left|x_{-}\right|+2\right)\left(1+\sqrt{n}\left(\left|v_{-}\right|+R^{\prime}\right)\right)}{\alpha(\alpha+1)\left(\frac{\left|v_{-}\right|}{\sqrt{2}}-R^{\prime}\right)^{2}(1-r)^{\alpha}} .
$$

Then we use the estimate (6.12) and the estimate (6.11) that gives an estimate on $\left|\dot{y}_{+}(t)\right|$ for $t \geq 0$, and we obtain

$$
\delta_{2} \leq \frac{2 n \beta_{2}\left(\delta_{2}+2\left|x_{-}\right|+3\right)\left(1+\sqrt{n}\left(\left|v_{-}\right|+R^{\prime}\right)\right)}{\alpha(\alpha+1)\left(\frac{\left|v_{-}\right|}{\sqrt{2}}-R^{\prime}\right)^{2}(1-r)^{\alpha}} .
$$


Using twice condition (2.16) one has

$$
\frac{1}{2} \delta_{2} \leq \frac{2 n \beta_{2}\left(2\left|x_{-}\right|+3\right)\left(1+\sqrt{n}\left(\left|v_{-}\right|+R^{\prime}\right)\right)}{\alpha(\alpha+1)\left(\frac{\left|v_{-}\right|}{\sqrt{2}}-R^{\prime}\right)^{2}(1-r)^{\alpha}} \leq \frac{r}{2} .
$$

Then $\delta_{2} \leq r$, and from (6.11) and (6.13) we have (2.25) and

$$
\delta_{2} \leq \frac{4 n \beta_{2}\left(\left|x_{-}\right|+2\right)\left(1+\sqrt{n}\left(\left|v_{-}\right|+R^{\prime}\right)\right)}{\alpha(\alpha+1)\left(\frac{\left|v_{-}\right|}{\sqrt{2}}-R^{\prime}\right)^{2}(1-r)^{\alpha}} .
$$

Estimate (2.24) follows from (6.15), (2.13) and the limit $y_{+}(t) \rightarrow 0$ as $t \rightarrow$ $+\infty$.

Note that similarly to $(6.6)$ one has for $(\varepsilon, \eta) \in[0,1]^{2}, \varepsilon \leq \eta$ and $\tau \geq 0$

$$
\begin{aligned}
\left.\mid z_{+}(a, \tau)+\eta x_{+}+\varepsilon y_{+}(\tau)\right) \mid & \geq\left|\eta x_{-}+\tau v_{-}\right|-\tau\left(\sup _{\mathbb{R}}\left|\dot{z}_{+}(a, .)-a\right|+\left|a_{s c}\right|\right)-\delta_{2} \\
& \geq \eta \frac{\left|x_{-}\right|}{\sqrt{2}}-r+\tau\left(\frac{\left|v_{-}\right|}{\sqrt{2}}-R^{\prime}\right)
\end{aligned}
$$

Note also that under condition (2.16) we have

$$
\max \left(\sup _{s \in(0,+\infty)} \max \left(1,1-r+\left(\frac{\left|v_{-}\right|}{\sqrt{2}}-R\right) s\right)\left|\dot{y}_{+}(s)\right|,\left(\frac{\left|v_{-}\right|}{\sqrt{2}}-R^{\prime}\right) \sup _{(0,+\infty)}\left|y_{+}\right|\right) \leq R .
$$

We now shorten $z_{+}(a,$.$) to z_{+}$for the rest of the paragraph. We also set $\varepsilon_{-}=0$ and $\varepsilon_{+}=1$. We need the following Lemma.

Lemma 6.1. Set

$$
\gamma_{ \pm}(t):=y_{ \pm}(t)-\int_{t}^{ \pm \infty} \int_{\tau}^{ \pm \infty}\left(B\left(z_{ \pm}(s)+x_{ \pm}\right)-B^{l}\left(z_{ \pm}(s)\right)\right) \dot{z}_{ \pm}(s) d s d \tau
$$

for $t \in \mathbb{R}$. Then the following estimates are valid

$$
\left|\dot{\gamma}_{ \pm}(t)\right| \leq \frac{n R \beta\left(1+\frac{1}{1-r}\right)\left(1+\frac{1+\sqrt{n}\left(\left|v_{-}\right|+R^{\prime}\right)}{\frac{\left|v_{-}\right|}{\sqrt{2}}-R^{\prime}}\right)+\sqrt{n} \beta_{2}\left(1+\sqrt{n}\left(\left|x_{-}\right|+\varepsilon_{ \pm}\right)\right)}{(\alpha+1)\left(\frac{\left|v_{-}\right|}{\sqrt{2}}-R^{\prime}\right)\left(1-r+\left(\frac{\left|v_{-}\right|}{\sqrt{2}}-R^{\prime}\right)|t|\right)^{\alpha+1}}
$$

for $\pm t \geq 0$

$$
\left|\dot{\gamma}_{-}(t)\right| \leq \frac{4 n \beta R\left(1+\frac{1}{1-r}\right)\left(1+\frac{1+\sqrt{n}\left(\left|v_{-}\right|+R^{\prime}\right)}{\left(\frac{\left|v_{-}\right|}{\sqrt{2}}-R^{\prime}\right)}\right)+2 \sqrt{n} \beta_{2}\left(1+\sqrt{n}\left|x_{-}\right|\right)}{(\alpha+1)\left(\frac{\left|v_{-}\right|}{\sqrt{2}}-R^{\prime}\right)(1-r)^{\alpha+1}},
$$

for $t \geq 0$. 
Proof of Lemma 6.1. Note that

$\dot{\gamma}_{-}(t)=\dot{A}\left(y_{-}\right)(t)-\dot{A}(0)(t)-\int_{-\infty}^{t}\left(\nabla V\left(z_{-}(\sigma)+x_{-}\right)-\nabla V^{l}\left(z_{-}(\sigma)\right)\right) d \sigma$, for $t \in \mathbb{R}$.

The term $\dot{A}\left(y_{-}\right)(t)-\dot{A}(0)(t)$ is estimated by (5.11) for $t \leq 0$. We use the growth properties of $V^{l}$ and $V^{s}$ (see (1.4) and (1.5)) and we use (4.16) $(" f=0 ")$ to estimate $\int_{-\infty}^{t}\left(\nabla V\left(z_{-}(s)+x_{-}\right)-\nabla V^{l}\left(z_{-}(s)\right)\right) d s, t \leq 0$. Then we obtain (6.19) for $\gamma_{-}$. To prove (6.19) for $\gamma_{+}$, we consider an identity similar to (6.21), and we use (6.16), and we repeat the proof of (5.11) with appropriate substitutions of " $\left(z_{-}, x_{-}, y_{-}\right)$" by " $\left(z_{+}, x_{+}, y_{+}\right)$", and we also estimate $\int_{t}^{+\infty}\left(\nabla V\left(z_{+}(s)+x_{+}\right)-\nabla V^{l}\left(z_{+}(s)\right)\right) d s, t \geq 0$, by using (6.16) and the growth properties of $V^{s}, V^{l}$ (we also use that $\left|b_{s c}\right| \leq 1$ ). For $t \geq 0$ we again use the identity (6.21), and we use the following estimate that follows from (2.22), (2.14) and (2.16):

$$
\alpha^{-1} \sup _{(0,+\infty)}\left|\dot{y}_{-}\right| \leq \frac{R}{(\alpha+1)(1-r)} .
$$

Then we repeat the proof of (5.13) where we use the bound (6.22) on $\sup _{(0,+\infty)}\left|\dot{y}_{-}\right|$ in the estimate similar to (5.12) with $F^{l}$ in place of $F^{s}$ and with " $\left(f_{1}, f_{2}\right)=$ $\left(y_{-}, 0\right)$ " (otherwise we only use the bound $\left\|y_{-}\right\| \leq R$ ). Thus we obtain (6.20).

We prove (2.26). First note that from (2.17) it follows that

$$
\Delta=\sum_{j=1}^{4} \Delta_{j},
$$

where we set

$$
\begin{gathered}
\Delta_{1}:=-\int_{-\infty}^{+\infty}\left(\nabla V(x(\tau))-\nabla V\left(\tau v_{-}+x_{-}\right)\right) d \tau \\
\Delta_{2}:=-\int_{-\infty}^{+\infty} B(x(\tau)) \int_{-\infty}^{\tau} \nabla V(x(\sigma)) d \sigma d \tau \\
\Delta_{3}:=\int_{-\infty}^{+\infty} \int_{-\infty}^{\tau}\left(B(x(\tau))(B(x(\sigma)) \dot{x}(\sigma))-B\left(\tau v_{-}+x_{-}\right)\left(B\left(\sigma v_{-}+x_{-}\right) v_{-}\right)\right) d \sigma d \tau \\
\Delta_{4}:=\int_{-\infty}^{+\infty}\left(B(x(\tau))-B\left(z_{-}(\tau)+x_{-}\right.\right. \\
\left.+\int_{-\infty}^{\tau} \int_{-\infty}^{s_{1}}\left(B\left(z_{-}\left(s_{2}\right)+x_{-}\right)-B^{l}\left(z_{-}\left(s_{2}\right)\right)\right) \dot{z}_{-}\left(s_{2}\right) d s_{2} d s_{1}\right) v_{-} d \tau
\end{gathered}
$$


We prove the estimates (6.28)-(6.32) given below that provide a bound for $\Delta_{i}, i=1, \ldots, 4$. Then we collect those bounds and the bounds obtained for $\sup _{(0,+\infty)}\left|\dot{\gamma}_{-}\right|, \sup _{(-\infty, 0)}\left|\dot{\gamma}_{-}\right|$and $\sup _{(-\infty, 0)}\left|\gamma_{-}\right|$(see Lemma 6.1), and we use the decomposition (6.23) and (2.13), and we obtain (2.26).

We use the growth properties of $V$ (see (1.4) and (1.5)) and we use (4.15) and (4.16), and we obtain

$$
\begin{aligned}
\left|\Delta_{1}\right| \leq & n \beta_{2} \int_{-\infty}^{+\infty} \frac{\left(|\tau|\left(\sup _{(0,+\infty)}\left|\dot{y}_{-}\right|+\sup _{\mathbb{R}}\left|\dot{z}_{-}-v_{-}\right|\right)+\sup _{(-\infty, 0)}\left|y_{-}\right|\right) d \tau}{\left(1+\frac{\left|x_{-}\right|}{\sqrt{2}}-r+|\tau|\left(\frac{\left|v_{-}\right|}{\sqrt{2}}-R^{\prime}\right)\right)^{\alpha+2}} \\
& +n \beta_{3}^{s} \int_{-\infty}^{+\infty} \frac{\left(|\tau|\left(\sup _{(0,+\infty)}\left|\dot{y}_{-}\right|+\sup _{\mathbb{R}}\left|\dot{z}_{-}-v_{-}\right|\right)+\sup _{(-\infty, 0)}\left|y_{-}\right|\right) d \tau}{\left(1+\frac{\left|x_{-}\right|}{\sqrt{2}}-r+|\tau|\left(\frac{\left|v_{-}\right|}{\sqrt{2}}-R^{\prime}\right)\right)^{\alpha+2}} \\
\leq & \frac{2 n R^{\prime}}{\left(\frac{\left|v_{-}\right|}{\sqrt{2}}-R^{\prime}\right)^{2}\left(1+\frac{\left|x_{-}\right|}{\sqrt{2}}-r\right)^{\alpha}}\left(\frac{\beta_{2}}{\alpha}+\frac{\beta_{2}+\beta_{3}^{s}}{(\alpha+1)\left(1+\frac{\left|x_{-}\right|}{\sqrt{2}}-r\right)}\right. \\
& \left.+\frac{\beta_{3}^{s}}{(\alpha+2)\left(1+\frac{\left|x_{-}\right|}{\sqrt{2}}-r\right)^{2}}\right) .
\end{aligned}
$$

We use (1.4), (1.5) and (4.16), and we obtain

$$
\begin{aligned}
\left|\Delta_{2}\right| & \leq n^{\frac{3}{2}}\left(\int_{\mathbb{R}} \sup _{j, k}\left|B_{j, k}(x(\tau))\right| d \tau\right)\left(\int_{\mathbb{R}} \sup _{k}\left|\frac{\partial V}{\partial x_{k}}(x(\sigma))\right| d \sigma\right) \\
& \left.\leq \frac{4 n^{\frac{3}{2}}}{\left(\frac{\left|v_{-}\right|}{\sqrt{2}}-R^{\prime}\right)^{2}\left(1+\frac{\left|x_{-}\right|}{\sqrt{2}}-r\right)^{2 \alpha}}\left(\frac{\beta_{1}}{\alpha}+\frac{\beta_{2}}{(\alpha+1)\left(1+\frac{\left|x_{-}\right|}{\sqrt{2}}-r\right)}\right)^{2}\right\}
\end{aligned}
$$

We use the identity

$$
\begin{aligned}
& B(x(\tau))(B(x(\sigma)) \dot{x}(\sigma))-B\left(\tau v_{-}+x_{-}\right)\left(B\left(\sigma v_{-}+x_{-}\right) v_{-}\right) \\
= & \left(B(x(\tau))-B\left(\tau v_{-}+x_{-}\right)\right)(B(x(\sigma)) \dot{x}(\sigma)) \\
& \left.+B\left(\tau v_{-}+x_{-}\right)\left(B(x(\sigma))-B\left(\sigma v_{-}+x_{-}\right)\right) \dot{x}(\sigma)\right) \\
+ & B\left(\tau v_{-}+x_{-}\right) B\left(\sigma v_{-}+x_{-}\right)\left(\dot{x}(\sigma)-v_{-}\right) \text {for }(\sigma, \tau) \in \mathbb{R}^{2},
\end{aligned}
$$

and we use, in particular, the growth properties of $B((1.4),(1.5))$ and (4.15) and (4.16), and we obtain

$$
\begin{aligned}
& \left|\Delta_{3}\right| \leq \frac{8 n^{\frac{5}{2}} R^{\prime}\left(\left|v_{-}\right|+R^{\prime}\right)}{\left(\frac{\left|v_{-}\right|}{\sqrt{2}}-R^{\prime}\right)^{3}\left(1+\frac{\left|x_{-}\right|}{\sqrt{2}}-r\right)^{2 \alpha}}\left(\frac{\beta_{2}}{\alpha}+\frac{\beta_{2}+\beta_{3}^{s}}{(\alpha+1)\left(1+\frac{\left|x_{-}\right|}{\sqrt{2}}-r\right)}+\frac{\beta_{3}}{(\alpha+2)\left(1+\frac{\left|x_{-}\right|}{\sqrt{2}}-r\right)^{2}}\right) \\
& \times\left(\frac{\beta_{1}^{l}}{\alpha}+\frac{\beta_{2}}{(\alpha+1)\left(1+\frac{\left|x_{-}\right|}{\sqrt{2}}-r\right)}\right)+\frac{4 n^{2} R^{\prime}}{\left(\frac{\left|v_{-}\right|}{\sqrt{2}}\right)^{2}\left(1+\frac{\left|x_{-}\right|}{\sqrt{2}}\right)^{2 \alpha}}\left(\frac{\beta_{1}^{l}}{\alpha}+\frac{\beta_{2}}{(\alpha+1)\left(1+\frac{\left|x_{-}\right|}{\sqrt{2}}\right)^{2}}\right)^{2}
\end{aligned}
$$


First, under condition (2.16), the function $\int_{-\infty}^{\tau} \int_{-\infty}^{s_{1}}\left(B\left(z_{-}\left(s_{2}\right)+x_{-}\right)-B^{l}\left(z_{-}\left(s_{2}\right)\right)\right) \dot{z}_{-}\left(s_{2}\right) d s_{2} d s_{1}$, $\tau \in \mathbb{R}$, belongs to $M_{R, r}$. Then we use the growth properties of $B,(4.15)$ and (4.16), and we obtain

$$
\begin{aligned}
\left|\Delta_{4}\right| \leq & n^{\frac{3}{2}}\left|v_{-}\right| \int_{\mathbb{R}} \frac{\sup _{(-\infty, 0)}\left|\gamma_{-}\right|+|\tau| \sup _{(0,+\infty)}\left|\dot{\gamma}_{-}\right|}{\left(1-r+\frac{\left|x_{-}\right|}{\sqrt{2}}+\left(\frac{\left|v_{-}\right|}{\sqrt{2}}-R^{\prime}\right)|\tau|\right)^{\alpha+2}} \\
& \times\left(\frac{\beta_{3}^{s}}{1-r+\frac{\left|x_{-}\right|}{\sqrt{2}}+\left(\frac{\left|v_{-}\right|}{\sqrt{2}}-R^{\prime}\right)|\tau|}+\beta_{2}\right) d \tau \\
\leq & \frac{2 n^{\frac{3}{2}}\left|v_{-}\right|}{\left(\frac{\left|v_{-}\right|}{\sqrt{2}}-R^{\prime}\right)\left(1-r+\frac{\left|x_{-}\right|}{\sqrt{2}}\right)^{\alpha}}\left(\frac{\sup _{(-\infty, 0)}\left|\gamma_{-}\right|}{1-r+\frac{\left|x_{-}\right|}{\sqrt{2}}}\left(\frac{\beta_{3}^{s}}{(\alpha+2)\left(1-r+\frac{\left|x_{-}\right|}{\sqrt{2}}\right)}+\frac{\beta_{2}}{\alpha+1}\right)\right. \\
& \left.+\frac{\sup _{(0,+\infty)}\left|\dot{\gamma}_{-}\right|}{\frac{\left|v_{-}\right|}{\sqrt{2}}-R^{\prime}}\left(\frac{\beta_{3}^{s}}{(\alpha+1)\left(1-r+\frac{\left|x_{-}\right|}{\sqrt{2}}\right)}+\frac{\beta_{2}}{\alpha}\right)\right) .
\end{aligned}
$$

It remains to prove (2.27). From (2.11), (2.12), and (2.2) at $t=0\left(A\left(y_{-}\right)=\right.$ $\left.y_{-}\right)$it follows by straightforward computations that

$$
\begin{aligned}
& b_{s c}=y_{-}(0)-y_{+}(0)=W^{l}+\int_{-\infty}^{0} \int_{-\infty}^{\sigma} F^{s}\left(. v_{-}+x_{-}\right)(\tau) d \tau d \sigma-\int_{0}^{+\infty} \int_{\sigma}^{+\infty} F^{s}\left(. v_{-}+x_{-}\right)(\tau) d \tau d \sigma \\
& +\int_{-\infty}^{0} \int_{-\infty}^{\sigma} B^{s}\left(\tau v_{-}+x_{-}\right) \int_{-\infty}^{\tau} B\left(s_{1} v_{-}+x_{-}\right) v_{-} d s_{1} d \tau d \sigma \\
& -\int_{0}^{+\infty} \int_{\sigma}^{+\infty} B^{s}\left(\tau v_{-}+x_{-}\right)\left(\int_{-\infty}^{\tau} B\left(s_{1} v_{-}+x_{-}\right) v_{-} d s_{1}\right) d \tau d \sigma \\
& +W^{l, s}+\sum_{j=1}^{7}\left(\Omega_{-, j}-\Omega_{+, j}\right),
\end{aligned}
$$

where we set

$$
\begin{gathered}
\Omega_{\mp, 1}=-\int_{\mp \infty}^{0} \int_{\mp \infty}^{\sigma}\left(\nabla V^{l}(x(\tau))-\nabla V^{l}\left(z_{\mp}(\tau)+x_{\mp}\right)\right) d \tau d \sigma \\
\Omega_{\mp, 2}:=-\int_{\mp \infty}^{0} \int_{\mp \infty}^{\sigma}\left(\nabla V^{s}(x(\tau))-\nabla V^{s}\left(\tau v_{-}+x_{-}\right)\right) d \tau d \sigma \\
\Omega_{\mp, 3}:=-\int_{\mp \infty}^{0} \int_{\mp \infty}^{\sigma} B^{s}(x(\tau)) \int_{-\infty}^{\tau} \nabla V\left(x\left(s_{1}\right)\right) d s_{1} d \tau d \sigma \\
\Omega_{\mp, 4}:=\int_{\mp \infty}^{0} \int_{\mp \infty}^{\sigma} \int_{-\infty}^{\tau}\left(B^{s}(x(\tau))\left(B\left(x\left(s_{1}\right)\right) \dot{x}\left(s_{1}\right)\right)-B^{s}\left(\tau v_{-}+x_{-}\right)\left(B\left(s_{1} v_{-}+x_{-}\right) v_{-}\right)\right) d s_{1} d \tau d \sigma
\end{gathered}
$$




$$
\begin{gathered}
\Omega_{\mp, 5}:=\int_{\mp \infty}^{0} \int_{\mp \infty}^{\sigma}\left(B^{s}(x(\tau))\right. \\
\left.-B^{s}\left(z_{-}(\tau)+x_{-}+\int_{-\infty}^{\tau} \int_{-\infty}^{s_{1}}\left(B\left(z_{-}\left(s_{2}\right)+x_{-}\right)-B^{l}\left(z_{-}\left(s_{2}\right)\right)\right) \dot{z}_{-}\left(s_{2}\right) d s_{2} d s_{1}\right)\right) v_{-} d \tau d \sigma, \\
\Omega_{\mp, 6}:=\int_{\mp \infty}^{0} \int_{\mp \infty}^{\sigma}\left(B^{l}(x(\tau))-B^{l}\left(z_{\mp}(\tau)+x_{\mp}\right.\right. \\
\left.\left.\quad+\int_{\mp \infty}^{\tau} \int_{\mp \infty}^{s_{1}}\left(B\left(z_{\mp}\left(s_{2}\right)+x_{\mp}\right)-B^{l}\left(z_{\mp}\left(s_{2}\right)\right)\right) \dot{z}_{\mp}\left(s_{2}\right) d s_{2} d s_{1}\right)\right) \dot{z}_{\mp}(\tau) d \tau d \sigma, \\
\Omega_{\mp, 7}:=\int_{\mp \infty}^{0} \int_{\mp \infty}^{\sigma}\left(\left(B^{l}(x(\tau))-B^{l}\left(z_{\mp}(\tau)+x_{\mp}\right)\right) \dot{y}_{\mp}(\tau)+B^{l}\left(z_{\mp}(\tau)+x_{\mp}\right) \dot{\gamma}_{\mp}(\tau)\right) d \tau d \sigma .
\end{gathered}
$$

We prove the estimates (6.41)-(6.49) given below that give a bound for $\Omega_{ \pm, j}, j=1 \ldots 7$. Then we collect those bounds and the bounds obtained for $\sup _{(0,+\infty)}\left|\dot{\gamma}_{-}\right|, \sup _{ \pm s \in(0,+\infty)}\left|\dot{\gamma}_{ \pm}(s)\right|$ and $\sup _{ \pm s \in(0,+\infty)}\left|\gamma_{ \pm}(s)\right|$ (see Lemma 6.1 ), and we use the decomposition (6.33), and we obtain (2.27) (we also use the estimate (2.13)). From the growth properties of $V^{s}, V^{l}, B^{s}, B^{l}(1.4)$ and (1.5), and from (4.15), (4.16) and (6.16), it follows that

$$
\begin{aligned}
\left|\Omega_{\mp, 1}\right| \leq & \frac{n \beta_{2} \sup _{s \in(0,+\infty)}\left|y_{\mp}(\mp s)\right|}{\alpha(\alpha+1)\left(\frac{\left|v_{-}\right|}{\sqrt{2}}-R^{\prime}\right)^{2}(1-r)^{\alpha}} \leq \frac{n \beta_{2} R}{\alpha(\alpha+1)\left(\frac{\left|v_{-}\right|}{\sqrt{2}}-R^{\prime}\right)^{3}(1-r)^{\alpha}} \\
\left|\Omega_{\mp, 2}\right| \leq & \frac{n \beta_{3}^{s} \sup _{(-\infty, 0)}\left|y_{-}\right|}{(\alpha+1)(\alpha+2)\left(\frac{v_{-} \mid}{\sqrt{2}}-R^{\prime}\right)^{2}(1-r)^{\alpha+1}} \\
& \left.+\int_{-\infty}^{0} \int_{-\infty}^{\sigma} \frac{n \beta_{3}^{s}|\tau|\left(\sup _{\mathbb{R}}\left|\dot{z}_{-}-v_{-}\right|+\sup _{(0,+\infty)}\left|\dot{y}_{-}\right| d \tau d \sigma\right.}{\left(1-r+\frac{\left|x_{-}\right|}{\sqrt{2}}+\left(\frac{\left|v_{-}\right|}{\sqrt{2}}-R^{\prime}\right)|\tau|\right)^{\alpha+3}}\right) \\
\leq & \frac{n \beta_{3}^{s} R^{\prime}}{(\alpha+1)\left(\frac{\left|v_{-}\right|}{\sqrt{2}}-R^{\prime}\right)^{3}\left(1-r+\frac{\left|x_{-}\right|}{\sqrt{2}}\right)^{\alpha}}\left(\frac{1}{(\alpha+2)\left(1-r+\frac{\left|x_{-}\right|}{\sqrt{2}}\right)}+\frac{1}{\alpha}\right) . \\
\left|\Omega_{\mp, 3}\right| \leq & \left(\varepsilon_{\mp}+1\right) n^{\frac{3}{2}} \beta_{2} \int_{-\infty}^{0} \int_{-\infty}^{\tau} \frac{d \tau d \sigma}{\left(1-r+\frac{\left|x_{-}\right|}{\sqrt{2}}+\left(\frac{\left|v_{-}\right|}{\sqrt{2}}-R^{\prime}\right)|\tau|\right)^{\alpha+2}} \\
& \left.\times \int_{-\infty}^{0}\left(\frac{\beta_{1}^{l}}{\left(1+\frac{\left|x_{-}\right|}{\sqrt{2}}+\left(\frac{\left|v_{-}\right|}{\sqrt{2}}-R_{0}\right)|\tau|\right)^{\alpha+1}}+\frac{\beta_{2}}{\left(1+\frac{\left|x_{-}\right|}{\sqrt{2}}+\left(\frac{\left|v_{-}\right|}{\sqrt{2}}-R_{0}\right)|\tau|\right)^{\alpha+2}}\right) d \tau\right) \\
\leq & \frac{\left(\varepsilon_{\mp}+1\right) n^{\frac{3}{2}} \beta_{2}}{\alpha(\alpha+1)\left(\frac{\left|v_{-}\right|}{\sqrt{2}}-R^{\prime}\right)^{3}\left(1-r+\frac{\left|x_{-}\right|}{\sqrt{2}}\right)^{2 \alpha}}\left(\frac{\beta_{1}^{l}}{\alpha}+\frac{\beta_{2}}{(\alpha+1)\left(1+\frac{\left|x_{-}\right|}{\sqrt{2}}\right)}\right) .
\end{aligned}
$$




$$
\begin{aligned}
& \left|\Omega_{\mp, 4}\right| \leq \frac{\left(\varepsilon_{\mp}+1\right) n^{\frac{5}{2}}\left(\left|v_{-}\right|+R^{\prime}\right) R^{\prime} \max \left(\beta_{2}, \beta_{3}^{s}\right)}{\alpha(\alpha+1)\left(\frac{\left|v_{-}\right|}{\sqrt{2}}-R^{\prime}\right)^{4}\left(1-r+\frac{\left|x_{-}\right|}{\sqrt{2}}\right)^{2 \alpha}} \\
& \times\left(\left(\beta_{1}^{l}+\frac{\alpha \beta_{2}^{s}}{(\alpha+1)\left(1-r+\frac{\left|x_{-}\right|}{\sqrt{2}}\right)}\right)\left(\frac{2}{\alpha}+\frac{1}{(\alpha+2)\left(1-r+\frac{\left|x_{-}\right|}{\sqrt{2}}\right)}\right)\right. \\
& \left.+\frac{\beta_{2}}{\alpha}+\frac{\beta_{2}^{l}+\beta_{3}^{s}}{(\alpha+1)\left(1-r+\frac{\left|x_{-}\right|}{\sqrt{2}}\right)}+\frac{\beta_{3}^{s}}{(\alpha+2)\left(1-r+\frac{\left|x_{-}\right|}{\sqrt{2}}\right)^{2}}\right) \\
& \left|\Omega_{-, 5}\right| \leq \frac{n^{\frac{3}{2}} \beta_{3}^{s}\left|v_{-}\right| \sup _{(-\infty, 0)}\left|\gamma_{-}\right|}{(\alpha+1)(\alpha+2)\left(\frac{\left|v_{-}\right|}{\sqrt{2}}-R^{\prime}\right)^{2}\left(1-r+\frac{\left|x_{-}\right|}{\sqrt{2}}\right)^{\alpha+1}}, \\
& \left|\Omega_{+, 5}\right| \leq \frac{n^{\frac{3}{2}} \beta_{3}^{s}\left|v_{-}\right|}{(\alpha+1)\left(\frac{\left|v_{-}\right|}{\sqrt{2}}-R^{\prime}\right)^{2}\left(1-r+\frac{\left|x_{-}\right|}{\sqrt{2}}\right)^{\alpha}}\left(\frac{\sup _{(-\infty, 0)}\left|\gamma_{-}\right|}{\alpha+2}+\frac{\sup _{(0,+\infty)}\left|\dot{\gamma}_{-}\right|}{\alpha\left(\frac{\left|v_{-}\right|}{\sqrt{2}}-R^{\prime}\right)}\right), \\
& \left|\Omega_{\mp, 6}\right| \leq \frac{n^{\frac{3}{2}} \beta_{2}\left(\left|v_{-}\right|+R^{\prime}\right) \sup _{s \in(0,+\infty)}\left|\gamma_{\mp}(\mp s)\right|}{\alpha(\alpha+1)\left(\frac{\left|v_{-}\right|}{\sqrt{2}}-R^{\prime}\right)^{2}(1-r)^{\alpha}}, \\
& \left|\Omega_{\mp, 7}\right| \leq \frac{n^{\frac{3}{2}} \beta_{2}\left(\frac{R^{2}}{\frac{|v-|}{\sqrt{2}}-R^{\prime}}+\sup _{s \in(0,+\infty)}\left(1-r+\left(\frac{\left|v_{-}\right|}{\sqrt{2}}-R^{\prime}\right)|s|\right)\left|\dot{\gamma}_{\mp}(\mp s)\right|\right)}{\alpha(\alpha+1)\left(\frac{\left|v_{-}\right|}{\sqrt{2}}-R^{\prime}\right)^{2}(1-r)^{\alpha}} .
\end{aligned}
$$

For the bound for $\Omega_{+, 6}$ in (6.48), we also used under condition (2.16) that the function $h(\tau)=\int_{\tau}^{+\infty} \int_{s_{1}}^{+\infty}\left(B\left(z_{+}\left(s_{2}\right)+x_{+}\right)-B^{l}\left(z_{+}\left(s_{2}\right)\right)\right) \dot{z}_{+}\left(s_{2}\right) d s_{2} d s_{1}$, $\tau \in(0,+\infty)$, satisfies the same estimates as $y_{+}$does in $(6.17)$.

Proof of Theorem 1.1. Note that for the vector valued function $W=\left(W_{1}, \ldots, W_{n}\right)$ defined by (2.18) we have

$$
\begin{aligned}
& \rho W_{j}(\rho \theta, x):= \\
& \sum_{k=1}^{n} \theta_{k} \int_{-\infty}^{+\infty} \int_{0}^{1}<\nabla B_{j, k}\left(\varepsilon z_{-}\left(\rho \theta, \frac{\tau}{\rho}\right)+(1-\varepsilon) \tau \theta+x\right. \\
& \left.+\frac{1}{\rho} \int_{-\infty}^{\tau} \int_{-\infty}^{\sigma}\left(B\left(z_{-}\left(\rho \theta, \frac{s}{\rho}\right)+x\right)-B^{l}\left(z_{-}\left(\rho \theta, \frac{s}{\rho}\right)\right)\right) \frac{\dot{z}_{-}\left(\rho \theta, \frac{s}{\rho}\right)}{\rho} d s d \sigma\right) \\
& \rho\left(z_{-}\left(\rho \theta, \frac{\tau}{\rho}\right)-\tau \theta\right)+\int_{-\infty}^{\tau} \int_{-\infty}^{\sigma}\left(B\left(z_{-}\left(\rho \theta, \frac{s}{\rho}\right)+x\right)-B^{l}\left(z_{-}\left(\rho \theta, \frac{s}{\rho}\right)\right)\right) \frac{\dot{z}_{-}\left(\rho \theta, \frac{s}{\rho}\right)}{\rho} d s d \sigma>d \varepsilon d \tau
\end{aligned}
$$


for $(\theta, x) \in T \mathbb{S}^{n-1}$ and $\rho \in(0,+\infty)$ and $j=1 \ldots n$. Then the limit (1.17) follows from (6.50), Lemma 4.1 and (2.26).

Note that for the vector valued function $W^{l, s}=\left(W_{1}^{l, s}, \ldots, W_{n}^{l, s}\right)$ defined by $(2.20)$, we have

$$
\begin{aligned}
& \rho^{2} W_{j}^{l, s}(\rho \theta, x):=\sum_{k=1}^{n} \theta_{k} \int_{-\infty}^{0} \int_{-\infty}^{\sigma}<\int_{0}^{1} \nabla B_{j, k}^{s}\left(\varepsilon z_{-}\left(\rho \theta, \frac{\tau}{\rho}\right)+(1-\varepsilon) \tau \theta+x\right. \\
& \left.+\varepsilon \rho^{-1} \int_{-\infty}^{\tau} \int_{-\infty}^{s_{1}}\left(B\left(z_{-}\left(\rho \theta, \frac{s_{2}}{\rho}\right)+x\right)-B^{l}\left(z_{-}\left(\rho \theta, \frac{s_{2}}{\rho}\right)\right)\right) \frac{\dot{z}_{-}\left(\rho \theta, \frac{s_{2}}{\rho}\right)}{\rho} d s_{2} d s_{1}\right) d \varepsilon, \\
& \rho\left(z_{-}\left(\rho \theta, \frac{\tau}{\rho}\right)-\tau \theta\right)+\int_{-\infty}^{\tau} \int_{-\infty}^{s_{1}}\left(B\left(z_{-}\left(\rho \theta, \frac{s_{2}}{\rho}\right)+x\right)-B^{l}\left(z_{-}\left(\rho \theta, \frac{s_{2}}{\rho}\right)\right)\right) \frac{\dot{z}_{-}\left(\rho \theta, \frac{s_{2}}{\rho}\right)}{\rho} d s_{2} d s_{1}>d \tau d \sigma \\
& +\sum_{k=1}^{n} \int_{-\infty}^{0} \int_{-\infty}^{\sigma} \frac{\dot{z}_{-, j}\left(\rho \theta, \frac{\tau}{\rho}\right)}{\rho}<\int_{0}^{1} \nabla B_{j, k}^{l}\left(z_{-}\left(\rho \theta, \frac{\tau}{\rho}\right)+x\right. \\
& +\varepsilon \rho^{-1} \int_{-\infty}^{\tau} \int_{-\infty}^{s_{1}} B^{s}\left(z_{-}\left(\rho \theta, \frac{s_{2}}{\rho}\right)+x\right) \frac{\dot{z}_{-}\left(\rho \theta, \frac{s_{2}}{\rho}\right)}{\rho} d s_{2} d s_{1} \\
& \left.+\rho^{-1} \int_{-\infty}^{\tau} \int_{-\infty}^{s_{1}}\left(B^{l}\left(z_{-}\left(\rho \theta, \frac{s_{2}}{\rho}\right)+x\right)-B^{l}\left(z_{-}\left(\rho \theta, \frac{s_{2}}{\rho}\right)\right)\right) \frac{\dot{z}_{-}\left(\rho \theta, \frac{s_{2}}{\rho}\right)}{\rho} d s_{2} d s_{1}\right) d \varepsilon, \\
& \int_{-\infty}^{\tau} \int_{-\infty}^{s_{1}} B^{s}\left(z_{-}\left(\rho \theta, \frac{s_{2}}{\rho}\right)+x\right) \frac{\dot{z}_{-}\left(\rho \theta, \frac{s_{2}}{\rho}\right)}{\rho} d s_{2} d s_{1}>d \tau d \sigma \\
& +\int_{-\infty}^{0} \int_{-\infty}^{\sigma} B^{l}\left(z_{-}\left(\rho \theta, \frac{\tau}{\rho}\right)+x\right) \int_{-\infty}^{\tau} B^{s}\left(z_{-}\left(\rho \theta, \frac{s_{1}}{\rho}\right)+x\right) \frac{\dot{z}_{-}\left(\rho \theta, \frac{s_{1}}{\rho}\right)}{\rho} d s_{1} d \tau d \sigma \\
& -\int_{0}^{+\infty} \int_{\sigma}^{+\infty} \sum_{k=1}^{n} \theta_{k} \int_{0}^{1}<\nabla B_{j, k}^{s}\left(\varepsilon z_{-}\left(\rho \theta, \frac{\tau}{\rho}\right)+(1-\varepsilon) \tau \theta+x\right. \\
& \left.+\varepsilon \rho^{-1} \int_{-\infty}^{\tau} \int_{-\infty}^{s_{1}}\left(B\left(z_{-}\left(\rho \theta, \frac{s_{2}}{\rho}\right)+x\right)-B^{l}\left(z_{-}\left(\rho \theta, \frac{s_{2}}{\rho}\right)\right)\right) \frac{\dot{z}_{-}\left(\rho \theta, \frac{s_{2}}{\rho}\right)}{\rho} d s_{2} d s_{1}\right) d \varepsilon, \\
& \rho\left(z_{-}\left(\rho \theta, \frac{\tau}{\rho}\right)-\tau \theta\right) \\
& \left.+\int_{-\infty}^{\tau} \int_{-\infty}^{s_{1}}\left(B\left(z_{-}\left(\rho \theta, \frac{s_{2}}{\rho}\right)+x\right)-B^{l}\left(z_{-}\left(\rho \theta, \frac{s_{2}}{\rho}\right)\right)\right) \frac{\dot{z}_{-}\left(\rho \theta, \frac{s_{2}}{\rho}\right)}{\rho} d s_{2} d s_{1}\right)>d \tau d \sigma \\
& -\int_{0}^{+\infty} \int_{\sigma}^{+\infty} \sum_{k=1}^{n} \frac{\dot{z}_{+, j}\left(a(\rho \theta, x), \frac{\tau}{\rho}\right)}{\rho} \int_{0}^{1}<\nabla B_{j, k}^{l}\left(z_{+}\left(a(\rho \theta, x), \frac{\tau}{\rho}\right)+b(\rho \theta, x)\right. \\
& +\varepsilon \rho^{-1} \int_{\tau}^{+\infty} \int_{s_{1}}^{+\infty} B^{s}\left(z_{+}\left(a(\rho \theta, x), \frac{s_{2}}{\rho}\right)+b(\rho \theta, x)\right) \frac{\dot{z}_{+}\left(a(\rho \theta, x), \frac{s_{2}}{\rho}\right)}{\rho} d s_{2} d s_{1}
\end{aligned}
$$




$$
\begin{aligned}
& +\rho^{-1} \int_{\tau}^{+\infty} \int_{s_{1}}^{+\infty}\left(B^{l}\left(z_{+}\left(a(\rho \theta, x), \frac{s_{2}}{\rho}\right)+b(\rho \theta, x)\right)-B^{l}\left(z_{+}\left(a(\rho \theta, x), \frac{s_{2}}{\rho}\right)\right)\right) \\
& \left.\frac{\dot{z}_{+}\left(a(\rho \theta, x), \frac{s_{2}}{\rho}\right)}{\rho} d s_{2} d s_{1}\right), \\
& \left.\int_{\tau}^{+\infty} \int_{s_{1}}^{+\infty} B^{s}\left(z_{+}\left(a(\rho \theta, x), \frac{s_{2}}{\rho}\right)+b(\rho \theta, x)\right)\right) \frac{\dot{z}_{+}\left(a(\rho \theta, x), \frac{s_{2}}{\rho}\right)}{\rho} d s_{2} d s_{1}>d \varepsilon d \tau d \sigma \\
& \quad+\int_{0}^{+\infty} \int_{\sigma}^{+\infty} B^{l}\left(z_{+}\left(a(\rho \theta, x), \frac{\tau}{\rho}\right)+x\right) \\
& \quad\left(\int_{\tau}^{+\infty} B^{s}\left(z_{+}\left(a(\rho \theta, x), \frac{\eta}{\rho}\right)+b(\rho \theta, x)\right) \frac{\dot{z}_{+}\left(a(\rho \theta, x), \frac{\eta}{\rho}\right)}{\rho} d \eta\right) d \tau d \sigma .
\end{aligned}
$$

for $(\theta, x) \in T \mathbb{S}^{n-1}$ and $\rho \in(0,+\infty)$ and $j=1 \ldots n$. Then the limit (1.18) follows from (6.51), Lemma 4.1 and (2.27).

\section{Acknowledgments}

This work was partially supported by French grant ANR-13-JS01-0006.

\section{References}

[1] J. Derezinski, C. GÉrard, Scattering theory of classical and quantum N-particle systems, Springer-Verlag, Berlin Heidelberg, 1997.

[2] I. M. Gel'fand, S. G. Gindikin, M. I. Graev, Integral geometry in affine and projective spaces, Itogi Nauki i Tekhniki, Sovr. Prob. Mat. 16, 53-226 (1980) (Russian).

[3] I. W. Herbst, Classical scattering with long range forces, Comm. Math. Phys. 35, 193-214 (1974).

[4] A. Jollivet, On inverse scattering at high energies for the multidimensional nonrelativistic Newton equation in electromagnetic field, J. Inverse Ill-Posed Probl. 17:(5), 441476 (2009).

[5] A. Jollivet, On inverse scattering at fixed energy for the multidimensional Newton equation in a non-compactly supported field, J. Inverse Ill-Posed Probl. 21:(6), 713-734 (2013). 
[6] A. Jollivet, Inverse scattering at high energies for the multidimensional Newton equation in a long range potential, to appear in Asympt. Anal., preprint 2013, ArXiv:1306.3638.

[7] A. JolLivet, Inverse scattering at high energies for classical relativistic particles in a long range electromagnetic field, to appear in Ann. Henri Poincaré (see ArXiv:1401.0182 for a long version, preprint 2013).

[8] L. D. Landau, E. M. Lifschitz, The Classical Theory of Fields Pergamon Press New York, 1971.

[9] M. Loss, B. ThalleR, Scattering of particles by long-range magnetic fields, Ann. Physics 176:(1), 159-180 (1987).

[10] F. Natterer, The Mathematics of Computerized Tomography, Stuttgart: Teubner and Chichester: Wiley, 1986

[11] R. G. Novikov, Small angle scattering and X-ray transform in classical mechanics, Ark. Mat. 37, 141-169 (1999).

[12] J. RADOn, Über die Bestimmung von Funktionen durch ihre Integralwerte längs gewisser Mannigfaltigkeiten. Ber. Verh. Sächs. Akad. Wiss. Leipzig, Math.-Nat. K1 69, 262-277 (1917).

[13] B. Simon, Wave operators for classical particle scattering. Comm. Math. Phys. 23, 37-48 (1971).

[14] K. YaJima, Classical scattering for relativistic particles, J. Fac. Sci., Univ. Tokyo, Sect. I A 29, 599-611 (1982). 